\title{
Serotonin Activates Overall Feeding by Activating Two Separate Neural Pathways in Caenorhabditis elegans
}

\author{
Bo-mi Song ${ }^{1}$ and Leon Avery ${ }^{2}$ \\ ${ }^{1}$ Department of Molecular Biology, University of Texas Southwestern Medical Center, Dallas, Texas 75390-9148, and ${ }^{2}$ Department of Physiology and \\ Biophysics, Virginia Commonwealth University, Richmond, Virginia 23298-0551
}

Food intake in the nematode Caenorhabditis elegans requires two distinct feeding motions, pharyngeal pumping and isthmus peristalsis. Bacteria, the natural food of $C$. elegans, activate both feeding motions (Croll, 1978; Horvitz et al., 1982; Chiang et al., 2006). The mechanisms by which bacteria activate the feeding motions are largely unknown. To understand the process, we studied how serotonin, an endogenous pharyngeal pumping activator whose action is triggered by bacteria, activates feeding motions. Here, we show that serotonin, like bacteria, activates overall feeding by activating isthmus peristalsis as well as pharyngeal pumping. During active feeding, the frequencies and the timing of onset of the two motions were distinct, but each isthmus peristalsis was coupled to the preceding pump. We found that serotonin activates the two feeding motions mainly by activating two separate neural pathways in response to bacteria. For activating pumping, the SER-7 serotonin receptor in the MC motor neurons in the feeding organ activated cholinergic transmission from MC to the pharyngeal muscles by activating the Gs $\alpha$ signaling pathway. For activating isthmus peristalsis, SER-7 in the M4 (and possibly M2) motor neuron in the feeding organ activated the $\mathrm{G}_{12} \alpha$ signaling pathway in a cell-autonomous manner, which presumably activates neurotransmission from M4 to the pharyngeal muscles. Based on our results and previous calcium imaging of pharyngeal muscles (Shimozono et al., 2004), we propose a model that explains how the two feeding motions are separately regulated yet coupled. The feeding organ may have evolved this way to support efficient feeding.

\section{Introduction}

The growing epidemic of obesity and eating disorders demands the study of regulatory mechanisms of food intake. Food intake is regulated by various environmental and internal inputs such as sensory factors, nutritional status, emotional processing, decision making, and learning (Berthoud, 2004; Zheng and Berthoud, 2008). Studying mutants whose food intake is altered under various conditions has greatly advanced our understanding of the regulatory mechanisms (Barsh and Schwartz, 2002).

Caenorhabditis elegans, the bacteria-eating roundworm, is an attractive model organism for studying regulation of food intake. Genetic manipulation is easy and the anatomy is simple (Schafer, 2005). Feeding behaviors in C. elegans have been well characterized and are amenable to quantitative analysis. Moreover, the molecules that control food intake are also at least partly conserved in C. elegans (Douglas et al., 2005; You et al., 2008; Kaun and Sokolowski, 2009).

The pharynx, a neuromuscular tube, is the feeding organ in $C$. elegans. The pharynx consists of eight muscles and 20 neurons of

\footnotetext{
Received April 25, 2011; revised Dec. 1, 2011; accepted Dec. 8, 2011.

Author contributions: B.-m.S. designed research; B.-m.S. performed research; L.A. contributed unpublished reagents/analytic tools; B.-m.S. and L.A. analyzed data; B.-m.S. and L.A. wrote the paper.

This work was supported by United States Public Health Service Grant HL46154 (L.A.). We thank C. Li, R. Komuniecki, K. Miller, S. Nurrish, and A. Fire for kindly providing us with strains and reagents. We thank the Caenorhabditis Genetic Center for strains. Some mutations were generated by the International C. elegans Gene Knockout Consortium (http://www.celeganskoconsortium.omrf.org). Some mutations were generated by the National Bioresource Project for the Experimental Animal "Nematode C. elegans" (http://www.shigen.nig.ac.jp/c.elegans/index.jsp).

Correspondence should be addressed to Bo-mi Song, Department of Molecular Biology, University of Texas Southwestern Medical Center, 6000 Harry Hines Boulevard, Dallas, TX 75390-9148. E-mail: bomi.song@gmail.com. DOI:10.1523/JNEUROSCI.2064-11.2012

Copyright $\odot 2012$ the authors $\quad 0270-6474 / 12 / 321920-12 \$ 15.00 / 0$
}

14 types. The five large pharyngeal muscles are connected by gap junctions and constitute the three functional parts of the pharynx, the corpus, the isthmus and the terminal bulb, which are arranged from anterior to posterior (see Fig. 5) (Albertson and Thomson, 1976). The corpus takes in food from the environment and accumulates the food in the anterior isthmus by its contraction and subsequent relaxation. The accumulated food is transported to the terminal bulb by a peristaltic movement of the posterior isthmus. The grinder in the terminal bulb crushes the food for nutrient absorption in the intestine. The pharyngeal muscles show two feeding motions, pumping and isthmus peristalsis (IP). Pumping is a synchronized contraction and subsequent relaxation of the corpus, the anterior isthmus, and the terminal bulb (see Fig. $5 A$ ). Isthmus peristalsis is a peristaltic movement of the posterior isthmus (see Fig. $5 B$ ).

Like other animals, C. elegans activates food intake by activating the feeding motions in response to food (bacteria) (Croll, 1978). Among the 14 types of pharyngeal neurons, only MC and M4 are essential for normal fast pumping and for isthmus peristalsis, respectively (Avery and Horvitz, 1987, 1989). However, the mechanisms by which the pharyngeal neurons activate feeding in response to bacteria are unknown. Here, we attempted to understand the mechanisms by studying the process by which serotonin activates feeding, for the following reasons: First, serotonin is a putative food signal that controls feeding in C. elegans. Serotonin mimics the effects of food in controlling multiple behaviors, including activating pharyngeal pumping (PP) (Horvitz et al., 1982). Serotonin increases pharyngeal pumping in response to bacteria (Sze et al., 2000). Second, serotonin activates 
pumping via a 5-HT type 7 receptor SER-7, which is expressed in multiple pharyngeal neurons including MC and M4 (Hobson et al., 2006). In fact, to activate pumping, serotonin requires cholinergic transmission from MC to the pharyngeal muscles (Raizen et al., 1995). Furthermore, SER-7 expression in M4 raises the possibility that serotonin also activates isthmus peristalsis.

\section{Materials and Methods}

\section{General methods and strains}

Except when stated otherwise, C. elegans was cultured at $19^{\circ} \mathrm{C}$ as described by Brenner (1974). All worms used were hermaphrodites. In the main text only, the gene name is shown. The following mutant alleles were used.

Null mutations. Null mutations were as follows: $\bmod -1$ (ok103) V, ser4(ok512) I, ser-1(ok512) III, ser-7(tm1325) X, eat-2(ad465) II, gsa1(pk75) I, acy-1 (pk1279) III, gpa-12(pk322) X, dgk-1(sy428) X, pkc-1(nj3) $V$, unc-13(s69), unc-31(e928), tph-1(mg280).

Hypomorphic mutations. Hypomorphic mutations were as follows: kin-2(ce179) X, cha-1 (p1152) IV.

Gain-of-function mutations. Gain-of-function mutations were as follows: gsa-1 (ce81gf) I, acy-1 (ce2gf) III, HS::gsa-1(Q227L), punc-17::gpa-12(Q205L), punc-17::rho-1(G14V), punc-17::pkc-1B(A160E).

The wild-type strain was N2 (Brenner, 1974), and the mutant strains used were MT9668: $\bmod -1$ (ok103) V; AQ866: ser-4(ok512) III; DA2100: ser-7(tm1325) X; DA1814: ser-1(ok345) X; KG421: gsa1(ce81gf) I; DA2245: gsa-1(ce81gf) I; ser-7(tm1325) X; DA2301: ser7(tm1325) $X$; nyIs80[pflp-21::gfp $(+)]$; DA2298: ser-7(tm1325) $X$; adIs2298[pflp-21::ser-7(+) pflp-21::gfp $(+)]$,eat-2(ad465) II; ser7(tm1325) X; adIs2298[pflp-21::ser-7(+) pflp-21::gfp(+)]; DA2247: gsa-1(ce81gf) I; ser-7(tm1325) X; adEx2247[pser-7b::gfp rol-6(d)]; DA2375: gsa-1(ce81gf) I; ser-7(tm1325) X; adEx2375[pser-7b:: ser-7(+) pser-7b::gfp rol-6(d)]; DA2297: ser-7(tm1325) X; adEx2297 [pser-7b::ser-7(+) pflp-21::gfp], ser-7(tm1325) X; adEx[pflp-2:: ser-7(+) pflp-21::gfp]; KG532: kin-2(ce179) X; DA2191: gsa-1(pk75) I/hT2(bli-4(e937) let(q782) qIs48[myo-2::gfp pes-10::gfp ges-1::gfp] I); +/hT2(III); KG761: acy-1(pk1279) III; ceEx108[myo-3::acy-1(+)]; DA2374: gsa-1(pk75) I/hT2(bli-4(e937) let(q782) qIs48[myo-2::gfp pes-10::gfp ges-1::gfp] I); +/hT2(III); kin-2(ce179) X; DA2374: gsa1(pk75) I/hT2(bli-4(e937) let(q782) qIs48[myo-2::gfp pes-10::gfp ges-1::gfp] I); +/hT2(III); adEx2374[pflp-21::gsa-1(+) pflp-21::gfp]; KG621: pkIs296[phsp-16::gsa-1(Q227L)]; KG518: acy-1(ce2gf) III; DA2246: adIs2246[punc-17::gpa-12(Q205L) pacr-2::gfp]; QT309: nzIs29[punc-17:rho-1G14V punc-122::gfp]; PS2627: dgk-1(sy428) X; NL594: gpa-12(pk322) X; QT253: nzEx72[punc-17:rho-1(G14VF25N) pttx-3::gfp], dgk-1(sy428) X, gsa-1(ce81gf) I; ser-7(tm1325) X; adEx[pser-7b::gpa-12(Q205L) pser-7b::gfp rol-6(d)]; DA2376: gsa1(ce81gf) I; ser-7(tm1325) X; adEx2376[pser-7b::rho-1(G14V) pser-7b::gfp rol6(d)]; PR1152: cha-1 (p1152) IV; DA465: eat-2 (ad465) II; DA2189: cha-1 (p1152) IV; kin-2(ce179) X, unc-13(s69) I; DA509: unc-31(e928) IV, unc-31(ALLELE) IV; nzEx72[punc-17:gpa-12(Q205L) pttx-3::gfp]; KP2753: pkc-1(nj3) V; P2841: nuIs131[Punc-17::pkc-1B(A160E)], pkc-1(nj3) V; nzEx72[punc-17:gpa-12 (Q205L) pttx-3:::gfp]; DA2378: unc-31(e928) IV; adEx2378[pser-7b::unc31(+) pflp-21::gfp]; DA2377: unc-31(e928) IV; adEx2377[pflp-21::gfp]; KG835: unc-31(e928) IV; ceEx117[prab-3::unc-31(+) prab-3::gfp pttx-3::gfp].

\section{Drug treatment and feeding assay}

To examine drug effects on feeding in the absence of bacteria, feeding rates were quantified from 3- to 5-h-old L1 larvae that had never been exposed to bacteria. The L1 stage was selected because it was the only stage in which the feeding assays on serotonin-treated worms or their controls could be performed. To isolate the serotonin effect on feeding rates, we removed food from the tested worms. In the absence of food, it was impossible to quantify isthmus peristalsis rates from later stage worms in our setup. Observation of isthmus peristalsis in the absence of food required high-power optics, and thus the tested worms had to be mounted on agarose pads. The mounted worms constantly moved and even small movements of the later stage worms made them out of focus. After collecting embryos by egg preparation, we incubated them on unseeded nematode growth medium (NGM) plates for $2 \mathrm{~h}$. Newly hatched
L1 larvae ( $0-2 \mathrm{~h}$ old $)$ were then transferred to unseeded NGM plates and incubated for $3 \mathrm{~h}$. The effect of each drug on the feeding rates was examined after $15 \mathrm{~min}$ incubation on $2 \%$ agarose pads containing the drug. Final concentrations of serotonin (H7752; Sigma-Aldrich) and forskolin (F6886; Sigma-Aldrich) in the pad were $20 \mathrm{~mm}$ and $115.4 \mu \mathrm{M}$, respectively. The pad in which forskolin was dissolved contained $0.25 \%$ ethanol and the controls for forskolin-treated worms were incubated with the same concentration of ethanol. The $0.25 \%$ ethanol did not affect feeding rates (data not shown). Feeding motions of each larva were observed using a Zeiss Axiophot microscope with a $63 \times$ objective. Two minute videos were taken from each larva with a Hitachi kP-160 CCD camera and digitized using Adobe Premiere, version 6.5, for quantification of feeding rates. Feeding rates were calculated by averaging two measures per each animal ( pumps per $55 \mathrm{~s}$ ).

\section{Feeding assay on bacteria}

Developmentally synchronized L1 larvae were cultured on Escherichia coli $\mathrm{HB} 101$ until adulthood (for $54 \mathrm{~h}$ at $19^{\circ} \mathrm{C}$ ). For this assay, we used adult worms instead of L1 larvae because of technical problems in quantifying the isthmus peristalsis rate. Observation of isthmus peristalsis is possible only with mounted L1 worms on the agarose pad under highpower optics or with adult worms on fluorescent bacteria in our setup. To perform the feeding assays on bacteria and on serotonin under the same conditions, we initially tried mounted L1 larvae on the pad, but it was impossible to control the amount of bacteria that were given to each worm. Thus, we detected isthmus peristalsis using fluorescent bacteria in adult worms. During isthmus peristalsis, bacteria that are taken up into the pharyngeal lumen by pumping pass through the lumen of the posterior isthmus. In larvae the amount of bacteria that passes through the posterior isthmus each time was too little to emit sufficient fluorescence to detect isthmus peristalsis. After 7-8 $\mathrm{h}$ of starvation at room temperature (RT), feeding rates of individual animals were quantified by counting pharyngeal contractions $2-5 \mathrm{~min}$ after the transfer to test food (mCherry-expressing HB101) at room temperature. The test food was prepared by seeding $10 \mu \mathrm{l}$ of mCherry-expressing HB101 culture $(\mathrm{OD}=$ 5.0) on NGM plate and incubating for $5.5 \mathrm{~h}$ at RT before the assay. For Figure $1 E, 100 \mu \mathrm{l}$ of mCherry-expressing HB101 culture was used to prepare test food because the $t p h-1$ null mutant would not stay on food that was prepared from $10 \mu \mathrm{l}$ of the culture. Isthmus peristalsis was recognized by the motion of the fluorescent bacteria. Feeding motions of individual animals were observed with a Zeiss Stemi SV11 Apo microscope with rhodamine fluorescence filters (excitation at $546 \mathrm{~nm}$ and emission at $610-675 \mathrm{~nm}$ ) to measure isthmus peristalsis rate as well as the pumping rate. The feeding rate of each animal (pumps per minute) was calculated by averaging the three measures from each animal (pumps per $30 \mathrm{~s}$ ) and subsequently by multiplying by 2 .

\section{Molecular biology and transgenic strain construction}

The truncated ser-7 promoter $(2.2 \mathrm{~kb})$ for expression in M4 and M2 (Hobson et al., 2003) [pser-7 forward (F): 5'-CAA ACA GGT AGA CAA TGT TGT AAA CTG TGA-3'; pser-7 reverse (R): 5' ${ }^{\prime}$ TTC ACC CCT CAG GCT GTG-3'] was cloned by PCR from wild-type (N2) genomic DNA. The $1.3 \mathrm{~kb}$ ser-7 cDNA (SER-7 cDNA F primer: $5^{\prime}$-CCCGGGATGGCCCGTGCAGTC-3'; SER-7 cDNA R primer: 5'-CCCGGGCTAGACGTCACTTGGTTCGT-3') (Hobson et al., 2003) was cloned by PCR from a cDNA pool that was reverse transcribed from N2 mRNA extracts. $p f l p-21$ and $p f l p-2$ were a kind gift from Dr. Chris Li (City University of New York, New York, NY). pser-7b and $p f l p-21$ were cloned into HindIIIBamHI-digested pPD96.52. ser-7 cDNA was cloned into the EcoRI site of the two vectors containing each of the promoters. gsa- 1 cDNA (GSA- 1 cDNA F primer: $5^{\prime}$-GAA TTC ATG GGG TGC GTC G; GSA-1 cDNA R primer: 5'-GAA TTC TTA TAG AAG CTC GTA CTG TCG TAG) was cloned by PCR from a wild-type (N2) cDNA library. After digestion with EcoRI, the cloned gsa- 1 cDNA was inserted into the EcoRI site of pPD96.42 containing the $f l p-21$ promoter. Drs. S. Nurrish (University College, London, UK) and K. Miller (Oklahoma Medical Research Foundation, Oklahoma City, OK) kindly provided cDNAs that encode rho- $1(G 14 \mathrm{~V})$ and unc-31, respectively. NheI-SpeI-digested rho-1(G14V) cDNA and AgeI-NheI-digested unc-31 cDNA were inserted into the XhoI site of pPD96.52 containing the ser-7b 
promoter by blunt-end ligation. The $p f l p-2:: s e r-7(+)$ rescue construct was generated by the PCR-fusion method (Hobert, 2002) using the following primers: pflp-2 A primer, 5'-TCTGTGTTCACTCTACCAGGAACTTTTCTCACTTTTTAATACATATTTTCATGAAC-3'; pflp-2 A' primer, 5' TCTGTGTTCACTCTACCAGGA-3'; pflp-2 B primer, 5'-GAGATATGTTGACTGCACGGGCCATGGTTTGCGACAATTGGTTTGGCAACG3'; SER-7 cDNA C primer, 5'-ATGGCCCGTGCAGTC-3'; pPD9575 3'UTR D primer, 5' -GGAAACAGTTATGTTTGGTATATTGGG-3'. To generate DA2297, DA2298, and ser-7;Ex[pflp-2::ser-7(+) pflp-21::gfp], germline transformation was performed with pser-7::ser7(+) (100 ng/ $\mu \mathrm{l}), p f l p-21:: \operatorname{ser}-7(+)(50 \mathrm{ng} / \mu \mathrm{l})$ or $p f l p-2:: \operatorname{ser}-7(+)(75 \mathrm{ng} / \mu \mathrm{l})$, and $p f l p-21:: g f p(50 \mathrm{ng} / \mu \mathrm{l})$ as a injection marker in $\operatorname{ser}-7(\mathrm{tm} 1325)$. For DA2298, the extrachromosomal array was integrated into the chromosome by gamma irradiation ( $6 \mathrm{krad})$. The integration line was outcrossed five times against ser-7(tm1325). For DA2374, a mixture of pflp-21::gsa-1(+) $(50 \mathrm{ng} / \mu \mathrm{l})$ and $p f l p-21:: g f p(50 \mathrm{ng} / \mu \mathrm{l})$ was germline transformed in wild-type worms. Then, transgenic males were crossed with DA2191 hermaphrodites. $\mathrm{F}_{1}$ males that carried pmyo-2::gfp and $p f l p-21:: g f p$ were selected and crossed with DA2191 hermaphrodites. After crossing, $\mathrm{F}_{1}$ hermaphrodites that carry pmyo-2::gfp and $p f l p-21:: g f p$ were placed on individual plates. After letting the hermaphrodites self, the plates in which all adult worms had green pharynxes were selected. DA2375 and DA2376 were generated by germline transformation of pser-7::ser-7(+) $(50 \mathrm{ng} / \mu \mathrm{l})$ or pser-7::rho-1 $(\mathrm{Gl} 4 \mathrm{~V})(15 \mathrm{ng} / \mu \mathrm{l})$ in DA2245. pser-7b::gfp $(88 \mathrm{ng} / \mu \mathrm{l})$ and rol-6(sd) (pRF4; $50 \mathrm{ng} / \mu \mathrm{l})$ were used as co-injection markers. For DA2377 and DA2378, pflp-21::gfp (50 $\mathrm{ng} / \mu \mathrm{l})$ was germline transformed with or without pser-7b::unc-31(+) $(50$ $\mathrm{ng} / \mu \mathrm{l})$ in $u n c-31(e 928)$.

\section{Video analysis and statistical testing}

To test the hypothesis that isthmus peristalsis is coupled to either the preceding pump or the following pump, we asked whether the interval between isthmus peristalsis and either of the pumps correlated with the interval between the two consecutive pumps that are flanked by isthmus peristalsis (see Fig. $1 \mathrm{~A}$ ) using Pearson's correlation test. As shown in Figure $1 D$, pumping rates of wild-type worms in response to serotonin were quite variable. Among 165 serotonin-treated worms, we selected 19 worms that pumped $<60$ times per minute and 20 worms that pumped $>60$ times per minute. From each of the $2 \mathrm{~min}$ videos of the serotonin-treated worms (for details, see above, Drug treatment and feeding assay), we selected two consecutive pumps between which a clear isthmus peristalsis occurred and measured the intervals between isthmus peristalsis and the preceding or following pump using Video Frame Capture program (4Media Software Studio). The interval between the two consecutive pumps decreases as pharyngeal pumping rate increases. If isthmus peristalsis is coupled to the preceding or following pump, the interval between isthmus peristalsis and the pump should be constant regardless of pharyngeal pumping rate (i.e., there should be no correlation between the interval between isthmus peristalsis and the pump and the interval between the two consecutive pumps). In contrast, if isthmus peristalsis and either of the pumps is not coupled, the two intervals should have a strong correlation.

Testing for effects on isthmus peristalsis is complicated by the fact that most treatments that affect isthmus peristalsis also affect pumping, and isthmus peristalsis depends nonlinearly on pumping. Therefore, to compare isthmus peristalsis rates in two datasets, we required that the pumping rates in those datasets overlap, and then asked whether isthmus peristalsis differed between datasets at the same pumping rate. The second question was answered by fitting parabolas to the data. Both datasets were simultaneously fit to the following model:

$$
y_{i}=a x_{i}+b x_{i}^{2}+D_{i}\left(c x_{i}+d x_{i}^{2}\right) .
$$

$x_{i}$ and $y_{i}$ are pumping and isthmus peristalsis rates for worm $i, D_{i}$ is an indicator (dummy) variable that is 0 if worm $i$ is in dataset 1 and 1 if worm $i$ is in dataset 2 , and $a, b, c$, and $d$ are the fitting parameters. If isthmus peristalsis differs between the two datasets, $(c, d)$ will differ from $(0,0)$. This was tested by calculating the $F$ statistic as follows:

$$
F_{2, n_{1}+n_{2}-2}=\frac{\frac{c^{2}}{\sigma_{c}^{2}}+\frac{d^{2}}{\sigma_{d}^{2}}-2 \rho \frac{c d}{\sigma_{c} \sigma_{d}}}{2\left(1-\rho^{2}\right)},
$$

where $c, d$ are the least-squares estimates of $c$ and $d ; \sigma_{c}, \sigma_{d}$, and $\rho$ are estimates of the SE of $c$, the SE of $d$, and the correlation between $c$ and $d$ from the linear regression; and $n_{1}, n_{2}$ are the numbers of points in datasets 1 and 2 .

The level of significance is the upper tail probability of a standard $F$ distribution with 2 and $n_{1}+n_{2}-2 \mathrm{df}$. [Since $c$ and $d$ are squared in computing $F$, any deviation from $(0,0)$ gives a positive $F$, so this is a one-tailed test.]

\section{Data presentation}

For clarity, no more than three datasets are presented in each ellipse plot. As a result, some results are repeated in more than one panel. The feeding rates of wild-type $D, E, G, I$, and $J ; 2, D$ and $F ; 3, D$ and $E$; and $4, A, C$, and $F$. The feeding rates of wild-type animals without drug treatment are shown in Figures 1, D and $E ; 2, B$ and $N$; and 3, $B$ and $C$. The feeding rates of wild-type animals in the presence of $7.5 \mu \mathrm{M}$ forskolin are shown in Figures $2 C$ and $4, B$ and $D$. The feeding rates of the ser- 7 null mutant in the presence of serotonin are shown in Figures $1 F$ and $2, I$ and $J$. The feeding rates of the $g s a-1(g f)$ mutant animals in the presence of serotonin are shown in Figures $1, H$ and $M$, and 3I. The feeding rates of the gsa$1(\mathrm{gf})$;ser-7 mutant animals in the presence of serotonin are shown in Figures $1 \mathrm{H}$ and $2 I$. The feeding rates of the $g s a-1(g f)$;ser- 7 control transgenic animals in the presence of serotonin are shown in Figures $1 \mathrm{M}$ and $3, H$ and $I$. The feeding rates of the ser- 7 control transgenic animals are shown in Figure $1 I-K$. The feeding rates of the ser-7; Is [pflp-21::ser-7(+)] transgenic animals are shown in Figure $1, I$ and $L$. The feeding rates of wild-type animals and the ser-7 control mutant animals in response to bacteria are shown in Figure $1 N-P$, and $O$ and $P$, respectively. The feeding rates of the gsa-1 null mutant animals in the presence of serotonin are shown in Figures 2, E, G, $H$, and $M$, and $3 F$. The feeding rates of the Is [unc-17::gpa-12(gf)] transgenic animals without drug treatment are shown in Figures $3 B$ and $4 G$. The feeding rates of the Is[punc-17:: rho-1(G14V)] and Is [punc-17::rho-1(G14VF25N)] transgenic animals in the absence of serotonin are shown in Figure 3, $C$ and $G$. The feeding rates of the cha-1 hypomorphic mutant animals in response to serotonin are shown in Figure 4, $A$ and $E$.

\section{Results}

\section{Isthmus peristalsis is coupled to the preceding pharyngeal pump}

To ask whether serotonin controls isthmus peristalsis as well as pumping, we compared the rates of the two feeding motions of L1 larvae in presence of serotonin with the rates in its absence. Serotonin significantly increased the frequencies of both pharyngeal pumping and isthmus peristalsis. However, the following observations suggested that isthmus peristalsis is coupled to the preceding pharyngeal pump: First, isthmus peristalsis never occurred in the absence of pumping. Second, isthmus peristalsis always occurred immediately after a pump, although not every pump was followed by isthmus peristalsis. (On average, isthmus peristalsis occurred every 3.4 pumps.) To test whether the two feeding motions are coupled, we measured the time interval between isthmus peristalsis and the preceding or following pump (Fig. 1A). As the rate of pumping increases, the interval between two consecutive pumps decreases. As a result, if pumping and isthmus peristalsis are not coupled, both the interval between peristalsis and pump and the variance of this interval should decrease as the rate of pumping increases. This is what we saw for the interval between peristalsis and the following pump (Fig. $1 C$; $\left.r_{(37)}=0.9998, p<0.0001\right)$. In contrast, if pumping triggers isthmus peristalsis, one might expect the interval and its variance to remain constant as pumping rate increases. In fact, the interval between peristalsis and the preceding pump was small ( $\sim 150 \mathrm{~ms})$ and unaffected by the increase in pharyngeal pumping rate. The SD of the interval was also small ( $\sim 50 \mathrm{~ms}$ ) and constant (Fig. $1 B$; $r_{(37)}=0.1854, p=0.27$ ), which suggests that isthmus peristalsis is indeed coupled to the preceding pharyngeal pump. 
A

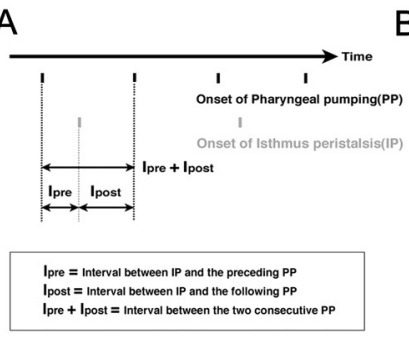

D

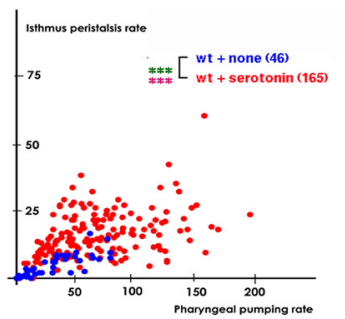

G

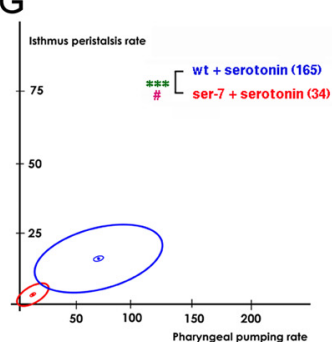

$\mathrm{J}$

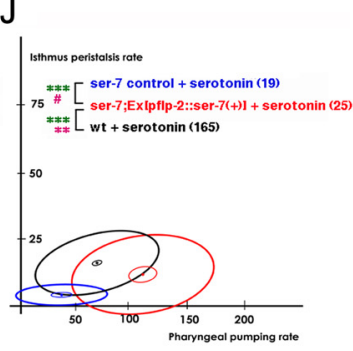

M

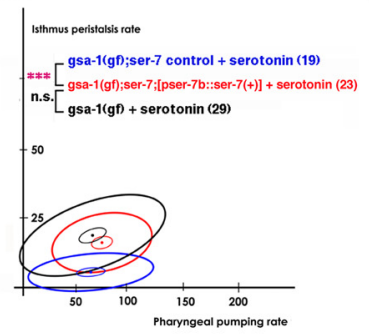

E

$\mathrm{H}$

K

$\mathrm{N}$
B
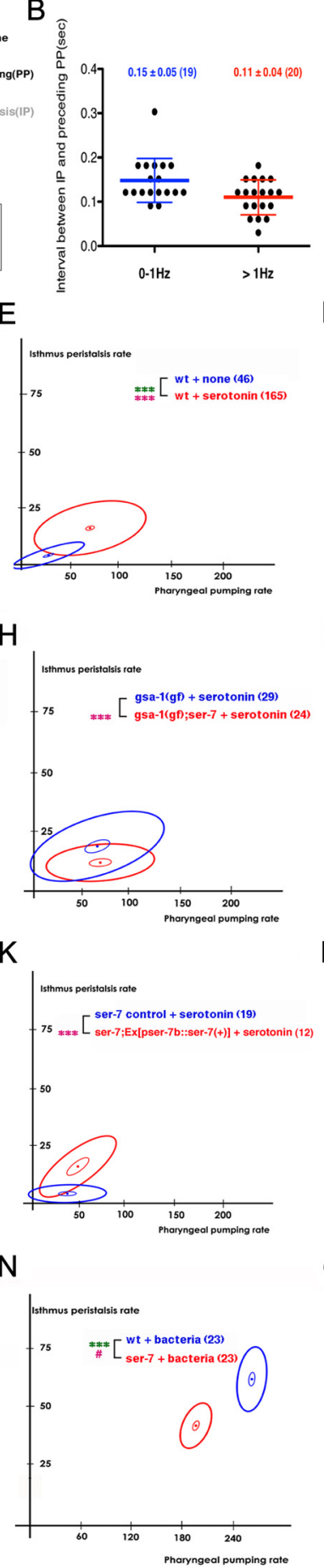

$\mathrm{P}$

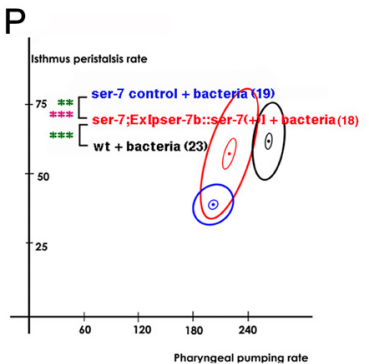

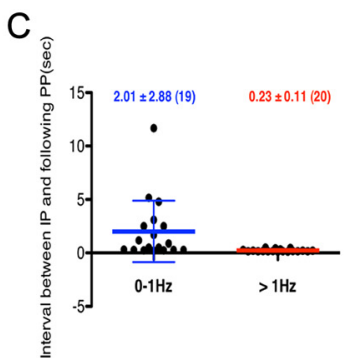

$\mathrm{F}$

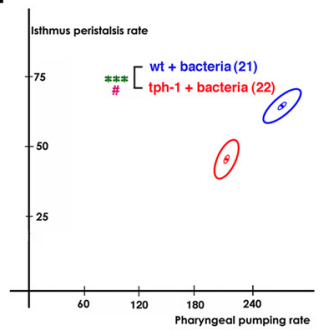

I

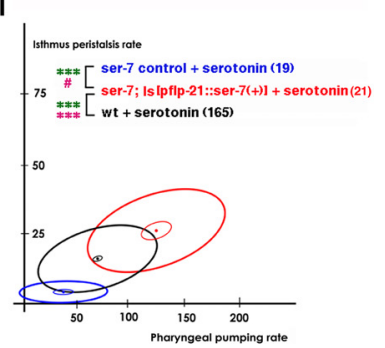

L

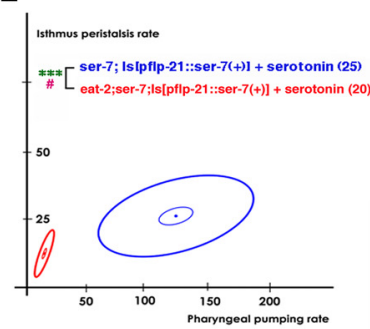

0

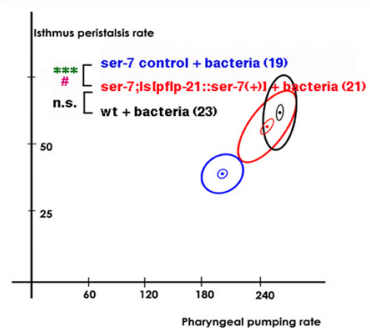

$B, C, I P$ was selectively coupled to the preceding $(\boldsymbol{B})$ but not to the following (C) PP in wild-type worms. Each filled circle indicates the interval between isthmus peristalsis and the indicated pump in one worm. ${ }^{* * *} p<0.001$; Pearson's correlation test; $n$.s. indicates not significant ( $p \geq 0.05$ ); data are shown as mean $\pm S D . \boldsymbol{D}, \boldsymbol{E}$, Serotonin activated both pumping and isthmus peristalsis. $\boldsymbol{D}$, A scatterplot of feeding rates of wild type (wt) with or without serotonin treatment. Each dot represents one worm. Thus, the $x$ and $y$ values of each dot indicate pumping rate and isthmus peristalsis rate, respectively, of one worm. $\boldsymbol{E}$, For simplicity, we transformed scatterplots into ellipse plots. The central dot is plotted at the mean pumping and isthmus peristalsis rate for each tested strain or condition. The large ellipse, a plot of root-mean-square deviation from the mean, shows the extent of variation from animal to animal. The width of the ellipse is $\sqrt{2}$ times the SD of pumping rate, the height is $\sqrt{2}$ times the SD of isthmus peristalsis rate, and narrowness or breadth is related to correlation. For normally distributed data, $63 \%$ of data points would be within the ellipse. The small ellipse similarly shows the likely range of the mean. $\boldsymbol{F}$, Both feeding rates of the $t p h-1(\mathrm{mg} 280)$ null mutant decreased to $75 \%$ of wild-type animals in response to bacteria, a physiologically relevant stimulant of feeding. $\mathbf{G}$, The ser7(tm 1325) null mutant was defective in activating pharyngeal pumping in response to serotonin. $\boldsymbol{H}$, The ser-7 null mutation decreased isthmus peristalsis rate in the gsa-1(ce81) gain-offunction mutant in response to serotonin. $I-M$, SER-7 in MC cell-autonomously activates pharyngeal pumping in response to serotonin. I, J, Expression of ser-7 (DNA using the flp-21 promoter (I) or the flp-2 promoter (J) fully restored pumping in the ser-7 null mutant in response to serotonin. pflp-2::ser-7(+) only partly restored isthmus peristalsis rate (J). This might be because the flp-2 promoter drives weak occasional expression in M4 (Kim and Li, 2004). $\boldsymbol{K}$, Expression of ser-7 CDNA in M4 using the ser-7b promoter increased isthmus peristalsis but not pumping in the ser-7 null mutant in response to serotonin. L, The eat-2(ad465) null mutation, which specifically blocks cholinergic transmission from MC to pharyngeal muscles, suppressed the rescue effect of pflp-21::ser-7(+) in the ser-7 mutant in response to serotonin. $\boldsymbol{M}$, SER-7 acts mainly in $M 4$ to stimulate isthmus peristalsis in response to serotonin. $\boldsymbol{N}$, Both feeding rates of the ser-7 null mutant decreased to $75 \%$ of wild-type animals in response to bacteria, a physiologically relevant stimulant of feeding. $\mathbf{0}$, As seen in response to serotonin, expression of ser-7 CDNA from the flp-21 promoter fully restored pumping in the ser-7 null mutant in response to bacteria. $\boldsymbol{P}$, Expression of ser-7 CDNA in M4 fully restored isthmus peristalsis rate in the ser-7 null mutant with a small effect on pharyngeal pumping in response to bacteria. ${ }^{* * *} p<0.001 ;{ }^{* *} p<0.01$; n.S., not significant $(p>0.05)$; unpaired $t$ test (two-tailed test) was used for comparison of pumping rates, and the level of significance is indicated by green asterisks; isthmus peristalsis rates were compared as described in Materials and Methods, and the level of significance is indicated by asterisks in pink. ${ }^{\text {} C o m-~}$ parison of isthmus peristalsis rates was infeasible due to lack of overlap in pumping rates between the two compared samples. The number of animals tested ( $n \geq 3$ independent experiments per each group) is shown in parentheses. For the comparisons with the transgenics expressing rescue constructs, transgenic animals expressing only co-injection marker(s) were used as controls.

Figure 1. Serotonin activates both pharyngeal pumping and isthmus peristalsis. SER-7 in MC and M4 (and possibly M2) separately activates pumping and isthmus peristalsis, respectively, in response to bacteria. Despite the separate regulation isthmus peristalsis is coupled to the preceding pump. $A$, A temporal representation of the onsets of pharyngeal pumping and isthmus peristalsis. Each black bar indicates onset of a pharyngeal pump. Each gray bar indicates the onset of an isthmus peristalsis. 
A
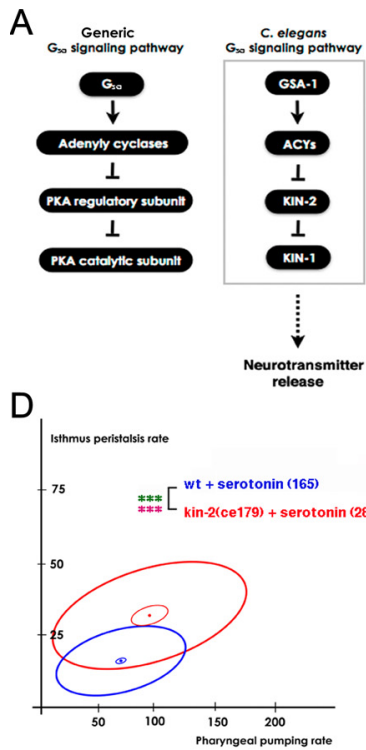

G

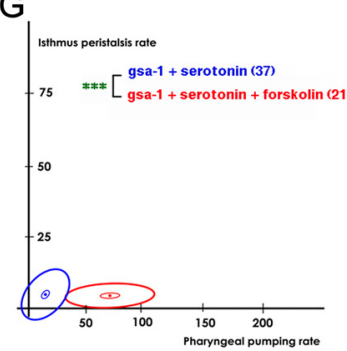

J

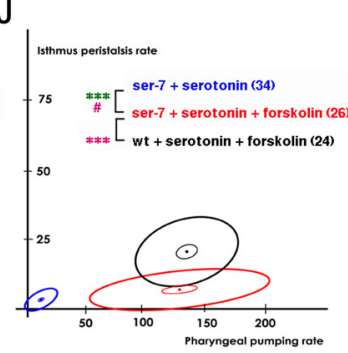

$\mathrm{M}$

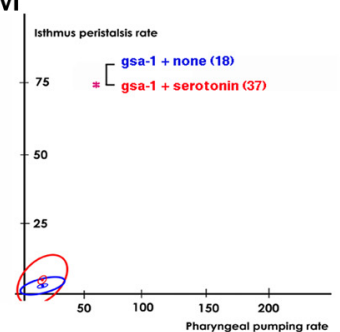

B

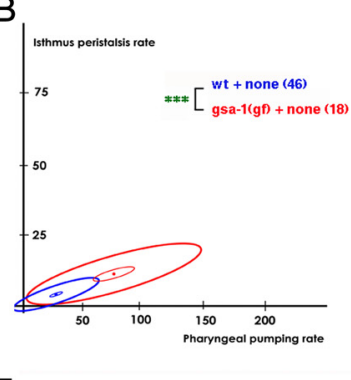

E

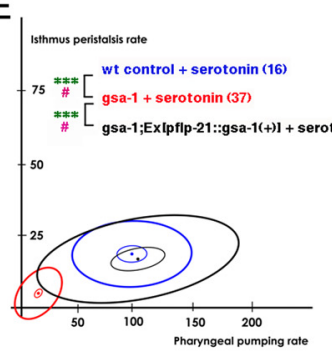

$\mathrm{H}$

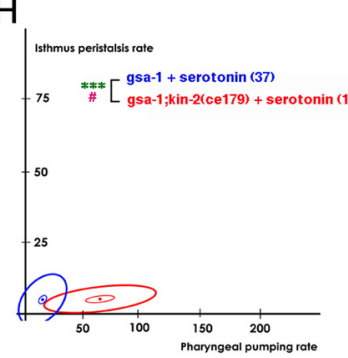

K

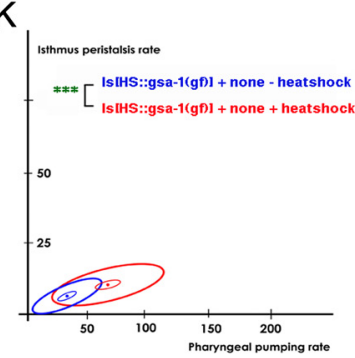

$\mathrm{N}$

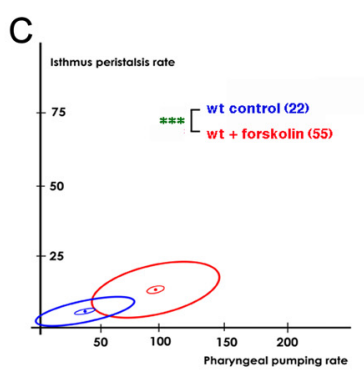

$\mathrm{F}$

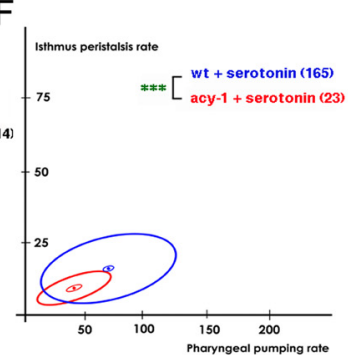

I

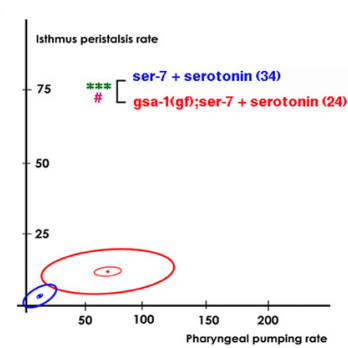

L

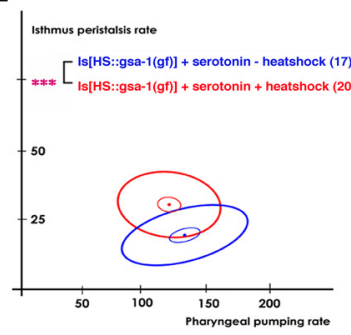

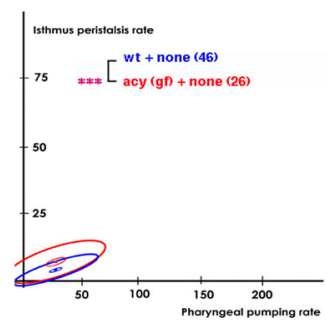

Figure 2. SER-7 activates pharyngeal pumping by activating the $G_{s} \alpha$ signaling pathway in response to serotonin. Activation of $\mathrm{G}_{\mathrm{s}} \alpha$ signaling has a small effect in activating isthmus peristalsis. $\boldsymbol{A}$, Schematic of the $\mathrm{G}_{\mathrm{s}} \alpha$ signaling pathway in C. elegans. $\boldsymbol{B}$, The constitutively active gsa-1 (ce81) mutation activated pumping as effectively as serotonin did. C, Activation of adenylyl cyclases by an acute forskolin treatment activated pumping as effectively as serotonin did. D, ce 179, a hypomorphic allele of kin-2, increased both feeding rates in response to serotonin. $\boldsymbol{E}$, The gsa-1(pk75) null mutation completely suppressed the serotonin-stimulated pharyngeal pumping. Expression of gsa-1 using the flp-21 promoter fully restored the pumping rate in the gsa-1(pk75) null mutant in response to serotonin. $\boldsymbol{F}$, An $a c y-1(p k 1279)$ null mutation attenuated pumping in response to serotonin. $\boldsymbol{G}, \boldsymbol{H}$, Forskolin $(\boldsymbol{G})$ and $c e 179(\boldsymbol{H})$, a hypomorphic allele of kin-2, increased pumping in the $g$ sa-1(pk75) null mutant in response to serotonin. $\boldsymbol{I}, \boldsymbol{J}$ Activation of $\mathrm{G}_{\mathrm{s}} \alpha$ signaling by constitutively active gsa-1(ce81) (I) or by forskolin treatment $(\boldsymbol{J})$ fully restored pumping in the ser-7(tm 1325) null mutant in response to serotonin. $\boldsymbol{K}$, Acute expression of constitutively active GSA-1(Q227L) using heat shock was sufficient to activate pharyngeal pumping, but not isthmus peristalsis in absence of serotonin. $L$, Acute expression of constitutively active GSA-1(Q227L) using heat shock activated both feeding motions in response to serotonin. $\boldsymbol{M}$, The gsa- 1 (pk75) null mutation failed to suppress serotonin-stimulated isthmus peristalsis. $\boldsymbol{N}$, The constitutively active $a c y-1$ (ce2) mutation activated

SER-7 serotonin receptor separately activates the two feeding motions mainly by activating pharyngeal neurons MC and M4

Since isthmus peristalsis is coupled to the preceding pharyngeal pump, the increased isthmus peristalsis rate in response to serotonin might be caused by the increased pharyngeal pumping rate. To find out whether serotonin stimulates isthmus peristalsis independent of pumping, we tested whether the isthmus peristalsis rate changed at a given pharyngeal pumping rate (for details of statistical analysis, see statistical analysis in Materials and Methods). To compare isthmus peristalsis rates at a given pharyngeal pumping rate, we used scatterplots in which the two feeding rates of each worm were plotted as a point $($ Fig. $1 D)$. In subsequent plots, we summarize the data as ellipses for clarity (for details, see Fig. $1 E$ legend). We found that serotonin stimulated isthmus peristalsis as well as pumping, and the effect on isthmus peristalsis was greater than what could be accounted for by the effect on pumping alone [Fig. $1 D, E ; t_{(209)}=6.8$, $p<0.0001$ (comparison of the pumping rates), $F_{(2,207)}=8.1, p=0.0004$ (comparison of the isthmus peristalsis rates)]. Concurrently, the frequencies of both pumping and isthmus peristalsis in the tph-1 null mutant decreased 25\% compared with wild type in response to bacteria (Fig. $1 F$; the pumping rates of wildtype and the tph-1 mutant animals were $266.1 \pm 3.0$ and $207.7 \pm 1.9$, respectively; the isthmus peristalsis rates of wild-type and the $t p h-1$ mutant animals were $65.5 \pm$ 0.9 and $46.6 \pm 1.0$, respectively). tph- 1 encodes a tryptophan hydroxylase required for serotonin biosynthesis (Sze et al., 2000). These data imply that serotonin directly activates both feeding motions in a coordinated manner.

We asked which receptor mediates the serotonin effect by examining the feeding rates of serotonin receptor null mutants in response to serotonin. Among five serotonin receptor null mutants, only the ser-7

$\leftarrow$

isthmus peristalsis but not pumping in the absence of serotonin. ${ }^{* * *} p<0.001 ;{ }^{*} p<0.05$; n.s., not significant $(p>$ 0.05 ); the unpaired $t$ test (two-tailed test) was used for comparison of pumping rates, and the difference indicated in green; isthmus peristalsis rates were compared as described in Materials and Methods, and the level of significance is indicated by asterisks in pink. "Comparison of isthmus peristalsis rates was infeasible due to lack of overlap in pumping rates between the two samples compared. The number of animals tested ( $n \geq 3$ independent assays per each group) is shown in parentheses. 
mutant failed to pump rapidly in presence of serotonin (Fig. $1 G$; data not shown; $t_{(197)}=8.4, p<0.0001$ ), confirming the previous report that SER-7 is the major serotonin receptor that mediates serotonin action on pumping (Hobson et al., 2006). On the contrary, it was not obvious which serotonin receptor activates isthmus peristalsis. The pharyngeal pumping rate of the ser-7 null mutant was too low to compare the isthmus peristalsis rate of the mutant with the rate of wild type (Fig. $1 G$ ) and the other serotonin receptor mutants had little or no change in isthmus peristalsis rate in response to serotonin (data not shown). To learn whether SER-7 affects isthmus peristalsis, we compared $g s a-1$ ( $g f)$ and $g s a-1(g f)$; ser-7 mutant animals. $g s a-1$ encodes $\mathrm{G}_{\mathrm{s}} \alpha$. The $g s a-1$ gain-of-function mutation was sufficient to activate pumping, even in the absence of SER-7 (Figs. $1 \mathrm{H}, 2 \mathrm{~B}$ ). Although $g s a-1(g f)$ and $g s a-1(g f)$; ser-7 pumped at the same rate in the presence of serotonin, the isthmus peristalsis rate was lower in $g s a-1(g f)$; ser-7 than in $g s a-1(g f)$ (Fig. $1 H ; F_{(2,49)}=10.7, p=0.0001$ ). These results suggest that serotonin signaling via SER-7 activates both feeding motions and that serotonin activates isthmus peristalsis separately from pharyngeal pumping.

We next asked in which cells SER-7 acts to activate the feeding motions. Based on previous cell ablation studies (Avery and Horvitz, 1987, 1989) and the expression pattern of SER-7 (Hobson et al., 2006), we hypothesized that SER-7 acts in MC and M4 to activate pharyngeal pumping and isthmus peristalsis, respectively. Consistent with the hypothesis that SER-7 in MC stimulates pumping, expression of SER-7 driven by the $f l p-21$ or the $f l p-2$ promoter fully restored serotonin-stimulated pumping in the ser-7 null mutant [Fig. 1 i; $t_{(53)}=14, p<0.0001$ (comparison of the pumping rates between the ser-7 and the ser-7; [pflp$21::$ ser-7 $(+)]$ mutant animals); Fig. $1 J ; t_{57}=13.2, p<0.0001$ (comparison of the pumping rates between the ser- 7 and the ser-7;[pflp-2::ser-7(+)] mutant animals); the pumping rates of the ser-7 control, the ser-7; [pflp-21::ser-7(+)], the ser-7; [pflp$2:: \operatorname{ser}-7(+)]$ and wild-type animals were $36.1 \pm 6.6,121.5 \pm 9.7$, $103.5 \pm 7.9$, and $67.5 \pm 3.0$, respectively]. The $f l p-21$ and the $f l p-2$ promoters drive expression in several neurons, and the expression patterns of $f l p-21$ and $f l p-2$ overlap only in MC and M4 (Kim and $\mathrm{Li}, 2004)$. Furthermore, expression of SER-7 in M4 using the $s e r-7 b$ promoter failed to change the pumping rate of the ser-7 null mutant in response to serotonin (Fig. $1 K$; the pumping rates of the ser-7 and the ser-7; [pser-7b::ser-7(+)] mutant animals were $36.1 \pm 6.6$ and $47 \pm 8.1$, respectively). The failure is unlikely to be due to insufficient expression because a significant increase in isthmus peristalsis rate was observed in the transgenic animals (Fig. $1 K ; F_{(2,27)}=33.1, p<0.0001$ ). To test whether SER-7 indeed acts mainly in MC to stimulate pumping, we tested whether blocking neurotransmission from MC blocks the rescue effect of $p f l p-21:: \operatorname{ser}-7(+)$ in the ser-7 mutant. EAT-2 is a nicotinic acetylcholine receptor subunit (Raizen et al., 1995). It is specifically localized in the pharyngeal muscles postsynaptic to MC (McKay et al., 2004), and thus an eat-2 null mutation selectively blocks cholinergic transmission from MC to pharyngeal muscles. An eat-2 null mutation suppressed the rescue effect of pflp-21::ser-7(+) (Fig. $1 L ; t_{39}=10.7, p<0.0001$ ), suggesting that SER-7 in MC activates pumping. Then, we tested whether SER-7 acts in M4 to stimulate isthmus peristalsis. The ser- $7 b$ promoter drives expression in M4 and occasionally in M2 (Hobson et al., 2003). As expected, expression of pser-7b::ser-7(+) rescue construct fully restored serotonin-stimulated isthmus peristalsis both in the ser-7 null mutant [Fig. $1 K ; F_{(2,27)}=33.1, p<0.0001$ (comparison of the isthmus peristalsis rates between the ser-7 control and the ser-7; [pser-7b::ser-7(+)] mutant animals); the isthmus peristalsis rates of the ser-7; $[$ pser-7b::ser-7(+)] mutant and wild-type animals were $15.8 \pm 2.7$ and $16 \pm 0.7$, respectively] and in the $g s a-1(g f)$;ser-7 mutant [Fig. $1 M ; F_{(2,38)}=11.1, p=$ 0.0002 (comparison of the isthmus peristalsis rates between the gsa-1(gf);ser-7 control and the $g s a-1(g f) ; s e r-7 ;[p s e r-7 b:: s e r-7(+)$ mutant animals); the isthmus peristalsis rates of the $g s a-1(g f)$; ser-7; [pser-7b::ser-7(+)] and the $g s a-1(g f)$ mutant animals were $16.0 \pm 1.6$ and $18.6 \pm 1.9$, respectively], supporting the hypothesis that SER-7 in M4 activates isthmus peristalsis.

Serotonin activates pharyngeal pumping in response to bacteria, the natural food of C. elegans (Fig. 1F) (Sze et al., 2000). Thus, we tested the physiological relevance of serotoninstimulated feeding by testing whether SER-7 in MC and M4 activates pharyngeal pumping and isthmus peristalsis, respectively, in response to bacteria. For this assay, we used adult worms for technical reasons (for details, see Materials and Methods, Feeding assay on bacteria). As expected, SER-7 was essential to fully activate isthmus peristalsis and pumping in response to bacteriapumping and isthmus peristalsis decreased 25\% compared with wild type in the ser-7 null mutant (Fig. $1 \mathrm{~N}$; the pumping rates of wild-type and the ser-7 mutant animals were $253.6 \pm 2.5$ and $189.7 \pm 2.6$, respectively; the isthmus peristalsis rates of wild-type and the ser- 7 mutant animals were $62.7 \pm 2.0$ and $42.8 \pm 1.4$, respectively). Consistent with our results from exogenous serotonin treatment, the $p f l p-21:: \operatorname{ser}-7(+)$ construct fully restored pumping and isthmus peristalsis rates to the wild-type level in response to bacteria (Fig. 1O; the pumping rates of wild-type and the ser-7; [pflp-21::ser-7(+) mutant animals were $253.6 \pm 2.5$ and $240.7 \pm 4.6$, respectively; the isthmus peristalsis rates of wild-type and the ser-7; $[p f l p-21:: \operatorname{ser}-7(+)]$ mutant animals were $62.7 \pm 2.0$ and $57.5 \pm 2.0$, respectively). In contrast, the pser-7b::ser-7(+) construct fully restored stimulated isthmus peristalsis with a small effect on pumping (Fig. $1 P$; the pumping rates of wild-type and the ser-7; [pser-7b::ser-7(+) mutant animals were $212 \pm 5.0$ and $194.9 \pm 3.4$, respectively; the isthmus peristalsis rates of wildtype and the ser-7;[pser-7b::ser-7(+)] mutant animals were $62.7 \pm 2.0$ and $58.3 \pm 3.9$, respectively). The small yet significant increase in pharyngeal pumping suggests that the two regulatory pathways that activate the feeding motions are not entirely distinct-one possible explanation is a mechanosensory effect of isthmus peristalsis on MC (Raizen et al., 1995). Together, these results suggest that SER-7 acts mainly in MC and M4 to stimulate pumping and isthmus peristalsis in response to serotonin, respectively.

\section{SER-7 cell-autonomously activates pharyngeal pumping via $\mathrm{G}_{\mathrm{s}} \boldsymbol{\alpha}$ signaling}

SER-7 is a type 7 serotonin receptor $\left(5-\mathrm{HT}_{7}\right)$. The $5-\mathrm{HT}_{7}$ receptor is a G-protein-coupled receptor that is thought to be coupled to $\mathrm{G}_{\mathrm{s}} \alpha$ and $\mathrm{G}_{12} \alpha$ (Kvachnina et al., 2005). In favor of the hypothesis that $\mathrm{G}_{\mathrm{s}} \alpha$ signaling is downstream of SER-7, heterologous expression of ser-7 cDNA increased intracellular cAMP level (Hobson et al., 2003), an indication of active $G_{s} \alpha$ signaling. Thus, we hypothesized that active SER-7 in MC and M4 increases feeding rates by activating $G_{s} \alpha$ and $G_{12} \alpha$ signaling pathways.

We first tested whether genetic manipulation of the conserved $\mathrm{G}_{\mathrm{s}} \alpha$ signaling pathway (Fig. $2 A$ ) (Reynolds et al., 2005) alters the feeding rate as the hypothesis predicts. GSA-1, ACY-1, and KIN-2 encode $\mathrm{G}_{\mathrm{s}} \alpha$, an adenylyl cyclase, and a regulatory subunit of protein kinase A (PKA), respectively (Fig. 2A). A constitutively active $g s a-1$ mutation increased pumping as effectively as serotonin did (Fig. $2 B ; t_{(62)}=5, p<0.0001$; the pumping rates of wild-type in the presence of serotonin and the $g s a-1(g f)$ mutant animals in 
A
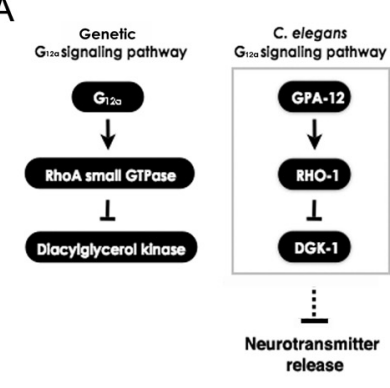

C

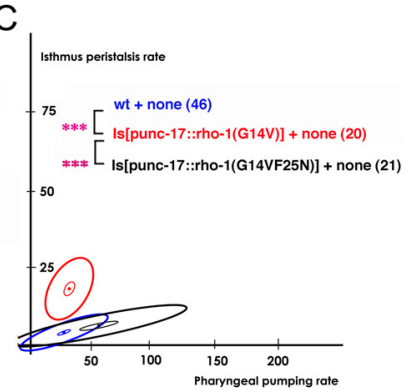

E

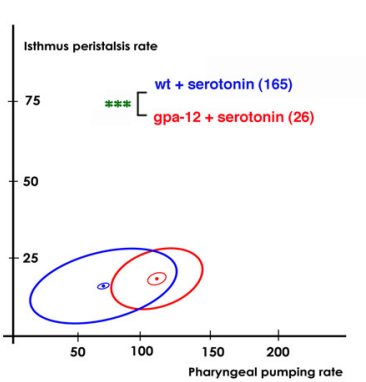

G
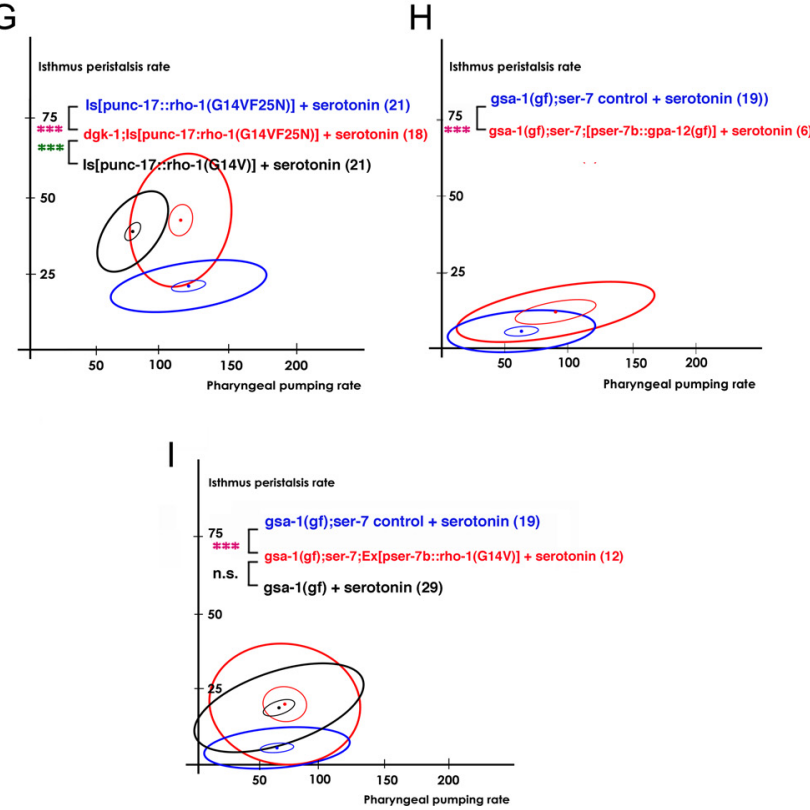

Figure 3. SER-7 activates isthmus peristalsis by activating $G_{12} \alpha$ signaling in $M 4$. $A$, Schematic of the $G_{12} \alpha$ signaling pathway in C. elegans. $B, C$, Expression of constitutively gpa$12(Q 205 L)(B)$ or constitutively active rho-1(G14V) (C) in cholinergic neurons was sufficient to activate isthmus peristalsis but not pharyngeal pumping in the absence of serotonin. $\boldsymbol{C}$, The F25N mutation completely suppressed the increase in isthmus peristalsis rate caused by the constitutively active rho-1(G14V) mutation. $\boldsymbol{D}$, The dgk-1(sy428) null mutation increased both feeding rates in response to serotonin. $\boldsymbol{E}, \boldsymbol{F}$, The $G_{12} \alpha$ and $G_{s} \alpha$ signaling pathways act redundantly to activate isthmus peristalsis in response to serotonin. The gsa-1(pk75); gpa-12(pk322) double-null mutant $(\boldsymbol{F})$, but not the gpa-12(pk322) single-null mutant $(\boldsymbol{E})$, decreased isthmus the absence of serotonin were $67.5 \pm 3.0$ and $74.1 \pm 12$, respectively). Furthermore, null mutations of $g s a-1$ and $a c y-1$ completely abolished and substantially attenuated activation of pumping, respectively (Fig. $2 E ; t_{(51)}=11.7, p<0.0001$ ) (Fig. $2 F$; $\left.t_{(185)}=3.3, p=0.001\right)$. We also found that an acute treatment with forskolin, an activator of adenylyl cyclases, activated pumping as effectively as serotonin did (Fig. $2 C ; t_{(75)}=6.6, p<0.0001$; the pumping rates of the serotonin-treated and the forskolintreated wild-type animals were $67.5 \pm 3.0$ and $92.3 \pm 4.9$, respectively). However, an $a c y-1$ gain-of-function mutation did not activate pumping (Fig. $2 \mathrm{~N}$ ), suggesting that adenylyl cyclases in addition to ACY-1 act together to activate pumping. cel79, a hypomorphic allele of kin-2, also increased pharyngeal pumping compared with wild type in presence of serotonin (Fig. 2D; $t_{(191)}=2.8, p=0.005$ ). cel79 by itself was not sufficient to activate pharyngeal pumping (data not shown), suggesting that the defective KIN-2 bearing the mutation still has some residual activity. In support of this hypothesis, ok248 and tm635, kin-2 null mutations cause larval lethality (International C. elegans Gene Knockout Consortium, http://www.celeganskoconsortium.omrf.org; National Bioresource Project of Japan, http://www.wormbase. org/db/misc/etree?name=WBPhenotype:0000062; class=Phenotype). Next, we confirmed the $G_{s} \alpha$ signaling pathway shown in Figure $2 \mathrm{~A}$. Both forskolin treatment (Fig. $2 G ; t_{(40)}=5.2, p<$ 0.0001 ) and $c e 179$ (Fig. $2 H ; t_{(50)}=7.4, p<0.0001$ ) in part restored the pumping rate in the $g s a-1$ null mutant, supporting the hypothesis that activated $\mathrm{G}_{\mathrm{s}} \alpha$ indeed acts by activating adenylyl cyclases and PKA in response to serotonin. Together, these data imply that activation of $\mathrm{G}_{\mathrm{s}} \alpha$ signaling is necessary and sufficient for serotonin to activate pharyngeal pumping.

Next, we tested whether $G_{s} \alpha$ signaling acts downstream of SER-7. If so, activation of the $\mathrm{G}_{\mathrm{s}} \alpha$ signaling pathway should bypass the ser-7 null mutation block in activating pumping in response to serotonin. As expected, both the constitutively active gsa-1 mutation (Fig. $2 I ; t_{(56)}=8.1, p<0.0001$ ) and forskolin treatment (Fig. $2 \mathrm{~J} ; t_{(43)}=7.6, p<0.0001$ ) fully restored pharyngeal pumping rate in the ser-7 null mutant. Although we cannot exclude the possibility that $\mathrm{G}_{\mathrm{s}} \alpha$ signaling acts in a pathway parallel to SER-7, these data support the hypothesis that $\mathrm{G}_{\mathrm{s}} \alpha$ signaling acts downstream of SER-7.

Finally, we asked whether SER-7 activates $\mathrm{G}_{\mathrm{s}} \alpha$ signaling in a cell-autonomous manner by testing whether expression of GSA-1 in flp-21-expressing cells [nine types of neurons including MC (Kim and Li, 2004)] is sufficient for normal serotoninstimulated pumping. Consistent with the hypothesis that SER-7 in $\mathrm{MC}$ cell-autonomously stimulates feeding, the rescue con-

peristalsis in response to serotonin. $\mathbf{G}, d g k-1$ (sy428) fully restored isthmus peristalsis rate in the transgenic animals expressing rho-1(F25NG14V) in cholinergic neurons to the level of the transgenics expressing rho-1(G14V) in response to serotonin. $\boldsymbol{H}$, Expression of constitutively active gpa-12(Q205L) in M4 (and occasionally in M2) significantly increased the isthmus peristalsis rate in gsa-1 (ce81); ser-7(tm 1325) in response to serotonin. I, Expression of constitutively active rho-1(G14V) in M4 (and occasionally in M2) fully restored isthmus peristalsis in gsa-1 (ce81);ser$7(t m 1325)$ in response to serotonin. The number of animals tested ( $n \geq 3$ independent assays per each group) is shown in parentheses. ${ }^{* * *} p<0.001 ;{ }^{*} p<0.05$; n.s., not significant $(p>$ 0.05 ); unpaired $t$ test (two-tailed test) was used for comparison of pharyngeal pumping rates, and the difference is marked in green; isthmus peristalsis rates were compared as described in Materials and Methods, and the level of significance is indicated by asterisks in pink; the number of animals tested ( $n \geq 3$ independent assays per each group) is shown in parentheses. For the comparisons with the transgenics expressing rescue constructs, the transgenic animals expressing only co-injection marker(s) were used as controls. 
struct fully restored pharyngeal pumping in the gsa- 1 null mutant (Fig. $2 E ; t_{(49)}=8.1, p<0.0001$; the pumping rates of the gsa-1;[pflp-21::gsa-1(+)] mutant and wild-type animals in presence of serotonin were $100 \pm 16.4$ and $67.5 \pm 3.0$, respectively).

Together, these data support the conclusion that SER-7 in MC activates pharyngeal pumping by activating the $\mathrm{G}_{\mathrm{s}} \alpha$ signaling pathway in a cell-autonomous manner in response to serotonin.

\section{SER-7 serotonin receptor in M4 activates isthmus peristalsis mainly by activating the $\mathrm{G}_{12} \alpha$ signaling pathway in a cell- autonomous manner}

Contrary to the dramatic and consistent effect on pumping, the $\mathrm{G}_{\mathrm{s}} \alpha$ signaling cascade had a relatively small effect on isthmus peristalsis. Constitutively active $a c y-1$ was sufficient to increase isthmus peristalsis rate, but the size of the increase was small (Fig. $\left.2 N ; F_{(2,67)}=10.6, p=0.0001\right) . k i n-2(c e 179)\left(\right.$ Fig. $2 D ; F_{(2,189)}=$ $35.4, p<0.0001)$ and acute expression of heat shock-inducible constitutively active $g s a-1\left(\mathrm{Q} 227 \mathrm{~L}\right.$ ) (Fig. $2 L ; F_{(2,33)}=8.3, p=$ 0.001 ) also significantly increased isthmus peristalsis rate in presence of serotonin. However, activation of no component of the $\mathrm{G}_{\mathrm{s}} \alpha$ signaling pathway by itself increased isthmus peristalsis rate as effectively as serotonin (Fig. $2 B, K, N$ ). Moreover, isthmus peristalsis in the gsa-1 null mutant increased in response to serotonin (Fig. $2 M ; F_{(2,51)}=4.6, p=0.01$ ), suggesting that an additional regulatory pathway exists. Since $5-\mathrm{HT}_{7}$ receptors also activate $G_{12} \alpha$ (Kvachnina et al., 2005), we hypothesized that $G_{s} \alpha$ and $\mathrm{G}_{12} \alpha$ act in parallel to increase isthmus peristalsis.

We first tested whether activation of the $\mathrm{G}_{12} \alpha$ signaling pathway increases isthmus peristalsis. gpa-12, rho-1, and $d g k-1$ encode $\mathrm{G}_{12} \alpha$, the small GTPase RhoA and diacylglycerol kinase components of the $\mathrm{G}_{12} \alpha$ signaling cascade, respectively (Fig. $3 A$ ) (Hiley et al., 2006; McMullan et al., 2006). In favor of the hypothesis, expression of constitutively active GPA-12 or RHO-1 in cholinergic neurons was sufficient to increase isthmus peristalsis rate (Fig. $3 B ; F_{(2,68)}=8.16, p=0.0007$ ) (Fig. $3 C ; F_{(2,62)}=78, p<$ $0.0001)$ in the absence of serotonin. A dgk-1 null mutation also increased the isthmus peristalsis rate greatly in the presence of serotonin (Fig. 3D; $F_{(2,183)}=35, p<0.0001$ ). Together, these data suggest that active $G_{12} \alpha$ signaling activates isthmus peristalsis.

The gpa-12 null mutation did not affect isthmus peristalsis rate in response to serotonin, suggesting that a redundant regulatory pathway exists (Fig. $3 E$ ). Since activation of $\mathrm{G}_{\mathrm{s}} \alpha$ signaling stimulated isthmus peristalsis, we speculated that $\mathrm{G}_{12} \alpha$ signaling and $\mathrm{G}_{\mathrm{s}} \alpha$ signaling pathways redundantly activate isthmus peristalsis. To test this possibility, we tested whether blocking both $\mathrm{G}_{\mathrm{s}} \alpha$ and $\mathrm{G}_{12} \alpha$ signaling pathways suppress the serotoninstimulated increase in isthmus peristalsis rate. Indeed, isthmus peristalsis rate was lower in the gsa-1; gpa-12 double-null mutant than in the gsa-1 single-null mutant (Fig. $3 F ; F_{(2,63)}=5.16, p=$ 0.008 ), confirming that $\mathrm{G}_{\mathrm{s}} \alpha$ and $\mathrm{G}_{12} \alpha$ signaling pathways redundantly stimulate isthmus peristalsis.

We next tested whether $G_{12} \alpha$ signaling activates isthmus peristalsis through the previously reported pathway (Fig. 3A). Hiley et al. (2006) and McMullan et al. (2006) reported that one role of RHO-1 is to activate neurotransmission downstream of $\mathrm{G}_{12} \alpha$ by directly binding to and inhibiting DGK-1 in C. elegans (Hiley et al., 2006; McMullan et al., 2006). We tested whether RHO-1 increases isthmus peristalsis by the same mechanism by testing whether F25N, a mutation of RHO-1 that disrupts the domain essential for DGK-1 inhibition, blocks the G14V (a constitutively active mutation)-induced increase in isthmus peristalsis rate. Indeed, punc-17::RHO-
1(G14VF25N) failed to increase isthmus peristalsis (Fig. 3C). Furthermore, the $d g k-1$ null mutation restored the isthmus peristalsis rate of the transgenics expressing punc-17::RHO1 (G14VF25N) to the rate of the transgenics expressing punc-17:: RHO-1(G14V) (Fig. 3G; the isthmus peristalsis rates of the [punc-17::RHO-1(G14V)] and dgk-1;[punc-17::RHO-1(G14VF25N)] transgenic animals were $37.8 \pm 2.0$ and $41.4 \pm 3.5$, respectively), suggesting that RHO-1 activates isthmus peristalsis by inhibiting DGK-1.

Finally, we tested whether SER-7 in M4 activates isthmus peristalsis by activating $G_{12} \alpha$ signaling in a cell-autonomous manner. For this, we asked whether expression of constitutively active GPA-12 or RHO-1 in M4 restores serotonin-stimulated isthmus peristalsis in the $g s a-1(g f) ;$ ser-7(tm1325) mutant animals. In support of our hypothesis, expression of the transgenes at least partially restored the isthmus peristalsis rates (Fig. $3 H ; F_{(2,21)}=6.3$, $p=0.007)$ (Fig. 3I; $F_{(2,27)}=8.7, p=0.001$ ) (comparison of the isthmus peristalsis rates between $g s a-1(g f) ; s e r-7$ control and gsa-1(gf);ser-7;[pser-7b::rho-1(G14V)] mutant worms). Together, these data support the hypothesis that SER-7 in M4 activates isthmus peristalsis rate by activating the $G_{12} \alpha$ signaling pathway in a cell-autonomous manner.

\section{The $\mathrm{G}_{\mathrm{s}} \boldsymbol{\alpha}$ signaling pathway activates pumping via cholinergic transmission from MC to the pharyngeal muscles}

Activation of either the $\mathrm{G}_{\mathrm{s}} \alpha$ signaling or the $\mathrm{G}_{12} \alpha$ signaling cascade increases neurotransmission in a cell-autonomous manner in C. elegans (Reynolds et al., 2005; Hiley et al., 2006; McMullan et al., 2006; Sieburth et al., 2007). To understand the mechanism by which MC and M4 activate the pharyngeal muscles to increase feeding rates in response to serotonin, we asked which neurotransmitters mediate the neural stimulation. We first confirmed the previous result (Raizen et al., 1995) that serotonin requires cholinergic transmission from $\mathrm{MC}$ to the pharyngeal muscles to increase pumping rate. A hypomorphic cha-1(p1152) mutation attenuated pumping in response to serotonin (Fig. 4A; $\left.t_{187}=4.6, p<0.0001\right)$. cha- 1 encodes choline acetyltransferase, an enzyme required for biosynthesis of acetylcholine, and $p 1152$ decreases choline acetyltransferase activity by $99 \%$ (Rand and Russell, 1984). Then, we confirmed that the cholinergic transmission from MC to the pharyngeal muscles stimulates pharyngeal pumping by testing whether an eat-2 null mutation suppresses the serotonin-stimulated pharyngeal pumping. Consistent with the previous report (Raizen et al., 1995), the eat-2 null mutation suppressed the serotonin-stimulated pumping (Fig. $4 C ; t_{(180)}=$ $5.5, p<0.0001$ ). Based upon our results suggesting that SER-7 activates pumping by activating $G_{s} \alpha$ signaling, we hypothesized that $\mathrm{G}_{\mathrm{s}} \alpha$ signaling activates pumping by activating cholinergic transmission from $\mathrm{MC}$ to the pharyngeal muscles. Indeed, the hypomorphic cha-1 (p1152) and eat-2 null mutations attenuated or completely suppressed the forskolin-stimulated pumping (Fig. $4 B ; t_{(71)}=4.9, p<0.0001$ ) (Fig. $4 D ; t_{(62)}=6.2, p<0.0001$ ), suggesting that the $\mathrm{G}_{\mathrm{s}} \alpha$ signaling pathway activated by SER-7 in $\mathrm{MC}$ increased pumping by activating acetylcholine release from $\mathrm{MC}$ to the pharyngeal muscles.

\section{$\mathrm{G}_{\mathrm{s}} \alpha$ and $\mathrm{G}_{12} \alpha$ signaling pathways activate isthmus peristalsis} via acetylcholine and neuromodulators packaged in dense core vesicles

Next, we tested whether M4 also activates isthmus peristalsis by releasing acetylcholine. We suspected acetylcholine for the following reasons: first, both M4 and M2 express genes necessary for cholinergic transmission (Alfonso et al., 1993). Second, acute treatment with arecoline, an activator of muscarinic acetyl- 
A

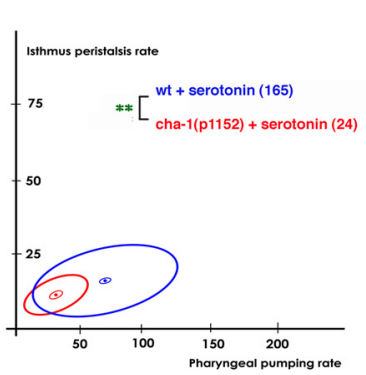

C

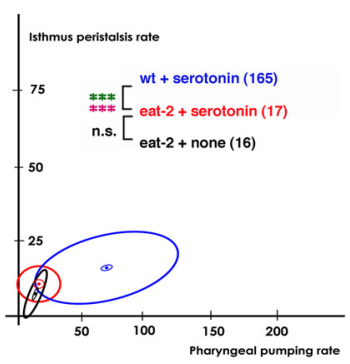

E

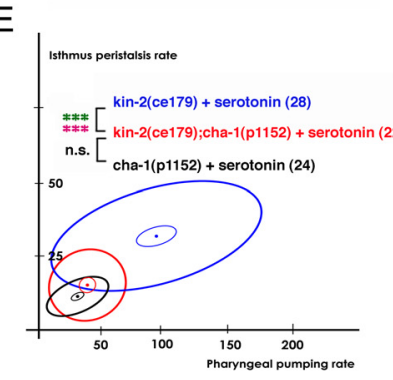

G
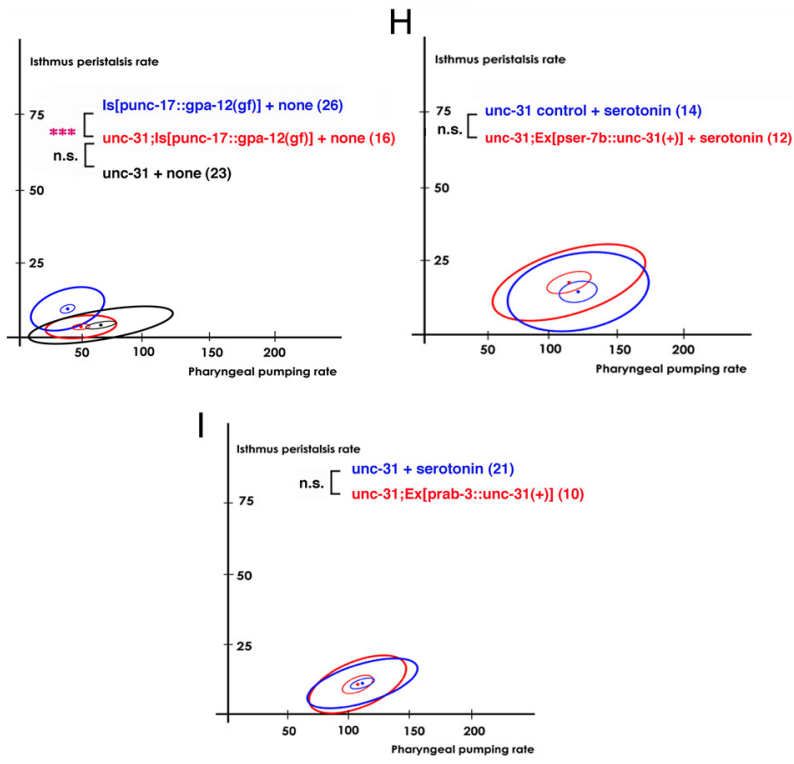

Figure 4. $G_{s} \alpha$ signaling activated pumping by activating acetylcholine transmission from $\mathrm{MC}$ to the pharyngeal muscles. $\mathrm{G}_{\mathrm{s}} \alpha$ signaling and $\mathrm{G}_{12} \alpha$ signaling activated isthmus peristalsis. Active isthmus peristalsis induced by $G_{s} \alpha$ signaling and $G_{12} \alpha$ signaling required the release of acetylcholine and dense core vesicles, respectively. $\boldsymbol{A}, \boldsymbol{B}$, The hypomorphic cha-1(p1152) mutation attenuated pumping in response to serotonin $(\boldsymbol{A})$ or forskolin $(\boldsymbol{B})$. $\boldsymbol{C}, \boldsymbol{D}$, The eat-2(ad465) null mutation suppressed the increase in pumping induced by serotonin $(\boldsymbol{C})$ or by forskolin $(\boldsymbol{D})$. $\boldsymbol{E}$, cha-1(p1152) attenuated the kin-2(ce179)-stimulated isthmus peristalsis. $\boldsymbol{F}$, The unc31 (e928) null mutation attenuated isthmus peristalsis rate in response to serotonin. $\boldsymbol{G}$, The unc-31(e928) null mutation completely suppressed the cholinergic GPA-12(Q205L)-stimulated isthmus peristalsis. $\boldsymbol{H}$, Expression of unc- $37 \mathrm{CDNA}$ in $\mathrm{M} 4$ failed to restore isthmus peristalsis rate in the unc-31(e928) mutant. $I$, Pan-neuronal expression of unc-31 cDNA failed to restore choline receptors, restored isthmus peristalsis in M4- worms (data not shown). We found that cha-1(p1152) attenuated the isthmus peristalsis rate in kin-2(ce179) (Fig. $4 E ; F_{(2,46)}=11.1$, $p=0.0001$ ) but not in wild type (Fig. $4 A$ ) in response to serotonin. This result could be explained in several ways. First, it might be that acetylcholine is indeed the major neurotransmitter by which M4 activates isthmus peristalsis, but the residual acetylcholine synthesized by the defective CHA- 1 barely supports the activation of isthmus peristalsis in wild-type animals. As a result, the effect of cha-1(p1152) is visible only in kin-2(ce179), where isthmus peristalsis occurs more frequently and thus more acetylcholine is required. Consistent with this possible explanation, we found no difference in the isthmus peristalsis rates between cha-1(p1152); kin-2(ce179) and cha-1(p1152) mutant animals (Fig. 4E).

Alternatively, but not mutually exclusively, M4 might activate isthmus peristalsis by releasing additional neurotransmitters. To test this possibility, we examined whether isthmus peristalsis rate is attenuated in $u n c-13$ and $u n c-31$ null mutants. Neurotransmitters are secreted from neurons through two types of vesicles, synaptic vesicles and dense core vesicles. Classical neurotransmitters such as GABA, acetylcholine, and glutamate are packaged in synaptic vesicles, whereas dense core vesicles contain neuropeptides and monoamines. unc-13 and unc-31 null mutations selectively yet completely block exocytosis of synaptic vesicles and dense core vesicles, respectively (Richmond et al., 2001; Zhou et al., 2007). The unc-13 null mutation result was not informative. The pharyngeal pumping rate of the unc-13 null mutant was too low to compare the isthmus peristalsis rate of the mutant with the rate of wild type. In contrast, the unc-31 null mutation substantially attenuated isthmus peristalsis in response to serotonin (Fig. $\left.4 F ; F_{(2,182)}=13.9, p<0.0001\right)$, suggesting that the neuromodulators packaged in dense core vesicles control isthmus peristalsis rate.

Next, we tested whether $G_{12} \alpha$ signaling activates isthmus peristalsis by releasing dense core vesicles. As expected, an unc-31 null mutation completely suppressed the increased isthmus peristalsis rate in the transgenics expressing constitutively active GPA- 12 in cholinergic neurons (Fig. 4G; $F_{(2,38)}=13.3, p<0.0001$ ). Since SER-7 and $\mathrm{G}_{12} \alpha$ signaling act in M4, we asked whether dense core vesicles released from M4 are responsible for activating isthmus peristalsis. However, expression of UNC-31 in M4 with a pser-7b::unc-31(+) transgene failed to restore isthmus peristalsis rate in the unc-31 mutant (Fig. $4 H$ ). Although we cannot exclude the possibility of insufficient expression, the failure of rescue is probably because UNC-31 acts in cells other than M4. GFP expressed from the co-injection marker $p f l p-21:: g f p$ was found in M4 neurons in all of the tested transgenic animals, which indirectly suggests expression of UNC-31 in M4 in the animals. Furthermore, pan-neuronal expression of UNC-31 also failed to restore serotonin-induced isthmus peristalsis in the unc-31 mutant (Fig. 4I) even though the expression rescued the locomotion phenotype (data not shown). From the rescue experiments, it is

$\leftarrow$

isthmus peristalsis rate in the unc-37(e928) mutant. The number of animals tested $(n \geq 3$ independent assays per each group) is shown in parentheses. ${ }^{* *} p<0.001$; ${ }^{* *} p<0.01$; n.s., not significant $(p>0.05)$; unpaired $t$ test (two-tailed test) was used for comparison of pharyngeal pumping rates, and the difference was marked in green; isthmus peristalsis rates were compared as described in Materials and Methods, and the level of significance is indicated by asterisks in pink; the number of animals tested ( $n \geq 3$ independent assays per each group) is shown in parentheses. For the comparisons with the transgenics expressing rescue constructs, the transgenic animals expressing only co-injection marker(s) were used as controls. 


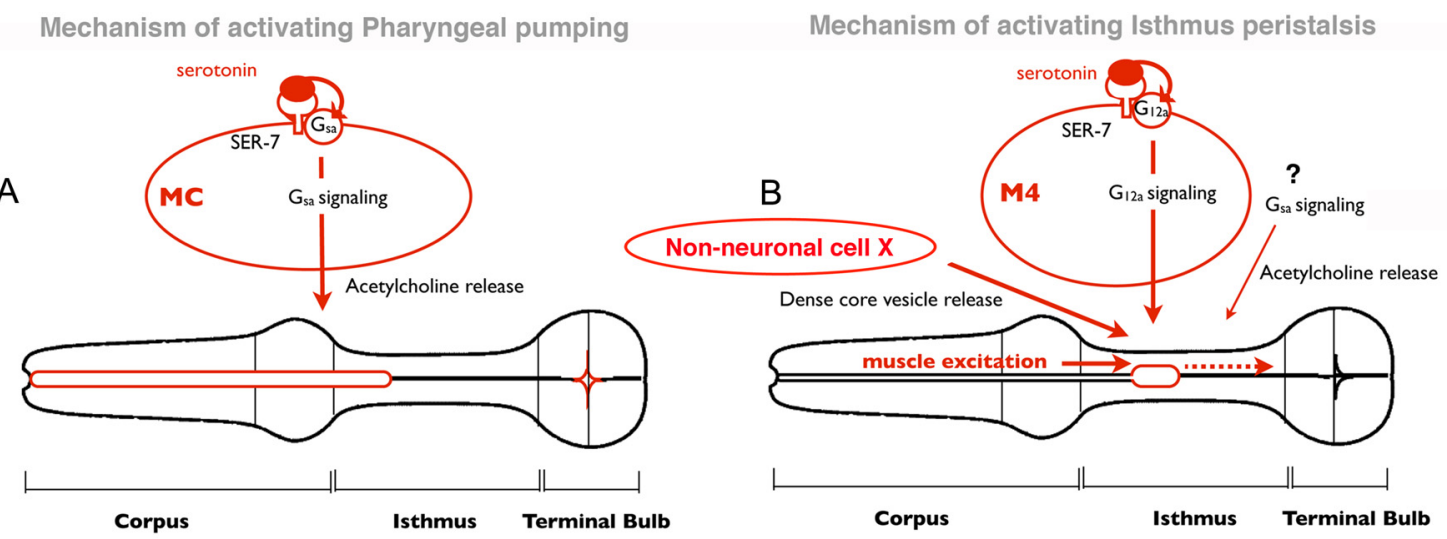

No Isthmus peristalsis without M4 stimulation

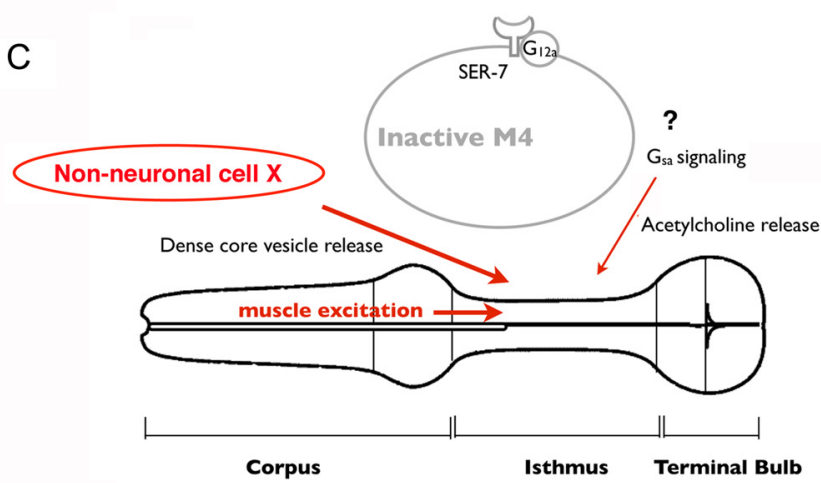

No Isthmus peristalsis without pharyngeal pumping

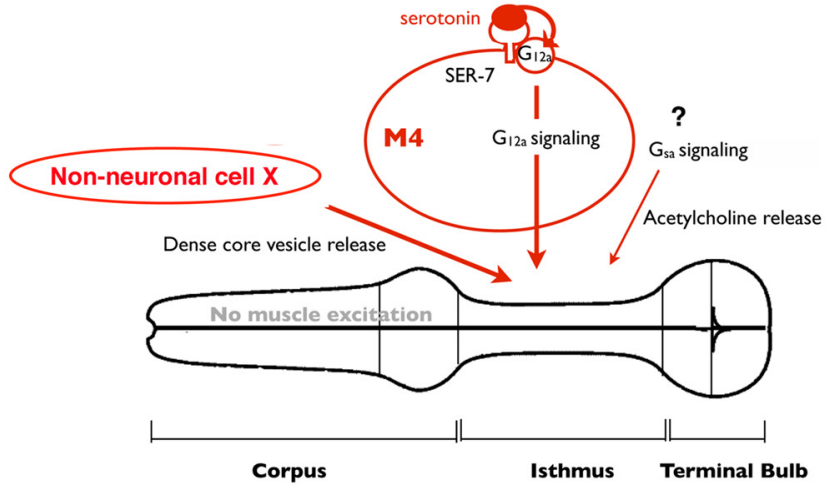

Figure 5. Model of regulation of feeding by serotonin. $A$, In response to serotonin, SER-7 in MC cell-autonomously activates its downstream $G_{s} \alpha$ signaling pathway, which subsequently stimulates pharyngeal pumping by activating cholinergic transmission from MC to the pharyngeal muscles. $\boldsymbol{B}$, In response to serotonin, SER-7 in M4 (and possibly in M2) activates its downstream $G_{12} \alpha$ pathways in a cell-autonomous manner, which subsequently activates $M 4$. The stimulus from active $M 4$, along with dense core vesicle release controlled by an unidentified pathway, activates isthmus peristalsis. The $G_{s} \alpha$ signaling pathway and downstream cholinergic transmission also contribute to activating isthmus peristalsis, but their sites of action have not been characterized. Given that expression of SER-7 in M4 fully restored isthmus peristalsis rate in the ser-7 null mutant, it is plausible that release of dense core vesicles from the unidentified cells is constitutively active rather than triggered by serotonin. C, Stimuli from an active M4 neuron and from the anterior part of the pharynx, excited by pumping, are both required to activate isthmus peristalsis. In the absence of either stimulus, isthmus peristalsis does not occur.

unclear whether M4 activates isthmus peristalsis via dense core vesicle release. However, given that an $u n c-31$ null mutation was epistatic to transgenic activation of $\mathrm{G}_{12} \alpha$ signaling (Fig. $4 G$ ), the dense core vesicle-releasing cells are either regulated by $\mathrm{M} 4$ or act in parallel with M4. Considering that M4 synapses directly onto the isthmus muscle, it is likely that UNC-31 acts in a parallel pathway (Fig. 5B). Together, these data imply that both releases of acetylcholine and dense core vesicles contribute to activating isthmus peristalsis. The release of acetylcholine is activated by the $\mathrm{G}_{\mathrm{s}} \alpha$ signaling pathway but the site of action is unidentified.
Which neurotransmitter M4 uses to activate isthmus peristalsis in response to serotonin is unclear, but dense core vesicle release from unknown non-neuronal cell(s) is essential in addition to the activation by M4.

\section{Discussion}

Here, we show that, in response to bacteria, serotonin activates the two feeding motions in C. elegans, pharyngeal pumping and isthmus peristalsis. Serotonin separately activates pumping and isthmus peristalsis mainly by activating SER-7 serotonin receptor 
in MC and M4 (and possibly M2), respectively. Activated SER-7 in $\mathrm{MC}$ and $\mathrm{M} 4$ activates neurotransmission mainly by activating $\mathrm{G}_{\mathrm{s}} \alpha$ and $\mathrm{G}_{12} \alpha$ signaling pathways, respectively, in a cellautonomous manner (Fig. $5 \mathrm{~A}, \mathrm{~B}$ ). The cholinergic transmission from $\mathrm{MC}$ to the pharyngeal muscles is the major trigger to activate pharyngeal pumping (Fig. $5 A$ ). In contrast, activation of isthmus peristalsis may require more than activation of M4 (Fig. $5 B$ ). Restoring SER-7 or activating RHO-1, a component of $\mathrm{G}_{12 \mathrm{a}}$ signaling pathway, in $\mathrm{M} 4$ fully restored isthmus peristalsis rate in mutants lacking ser-7 (Figs. $1 K, M, 3 I$ ), supporting that M4 is the major neuron by which serotonin activates isthmus peristalsis. However, restoring UNC-31 (required for serotonin-stimulated isthmus peristalsis) in M4 failed to restore isthmus peristalsis in the unc-31 null mutant (Fig. $4 \mathrm{H}$ ). Although it is still possible that M4 stimulates isthmus peristalsis by releasing dense core vesicles, failure to restore serotonin-induced isthmus peristalsis by panneuronal expression of UNC-31 (Fig. 4I) suggests that activating isthmus peristalsis requires dense core vesicle release from unidentified non-neuronal cells in addition to M4 activation. Considering SER-7 mainly acts in M4 to activate isthmus peristalsis in response to serotonin, it is possible that secretion of dense core vesicles from the unidentified cells may be constitutively active rather than being triggered by serotonin. The $\mathrm{G}_{\mathrm{s}} \alpha$ pathway, which contributes to the stimulation of isthmus peristalsis via cholinergic transmission (Fig. 5B), may act cell-autonomously downstream of SER-7 in M4 in parallel with the $G_{12} \alpha$ pathway, but the possibility has not been tested (Fig. 5C).

Our discovery that serotonin activates MC and M4 by mechanisms involving $G_{\mathrm{s}} \alpha$ and $\mathrm{G}_{12} \alpha$ signaling pathways, together with previous studies (Reynolds et al., 2005; McMullan et al., 2006), suggests that the mechanism by which G-protein signaling pathways increase neuronal activity is widely conserved throughout the nervous system in C. elegans.

How do $\mathrm{G}_{\mathrm{s}} \alpha$ and $\mathrm{G}_{12} \alpha$ signaling cascades activate neurotransmission? Several studies (Aravamudan and Broadie, 2003; Reynolds et al., 2005; McMullan et al., 2006) suggested that PKA and diacylglycerol, downstream of $\mathrm{G}_{\mathrm{s}} \alpha$ and $\mathrm{G}_{12} \alpha$ signaling cascades (Jose and Koelle, 2005), enhance synaptic efficacy by increasing the abundance of UNC-13 at the neuromuscular junction. UNC-13 is a primary determinant of synaptic vesicle priming and neurotransmitter release probability. However, several observations suggest that $\mathrm{G}_{\mathrm{s}} \alpha$ signaling in $\mathrm{MC}$ may activate pumping by a different mechanism that increases the firing rate (Raizen and Avery, 1994). An MC-dependent depolarization of pharyngeal muscle precedes every pump during normal fast pumping. Additionally, incubation of the pharynx with nicotine, an agonist of the nicotinic acetylcholine receptor, increased the duration but not frequency of pharyngeal contractions, suggesting that MC increases the pumping rate by increasing its firing rate. To increase pumping rate in response to serotonin, $\mathrm{G}_{\mathrm{s}} \alpha$ signaling may increase the firing rate of MC by modulation of ion channel activity. In fact, we observed that a null mutation of $\operatorname{shl}-1$, a voltage-gated A-type potassium channel (Fawcett et al., 2006), dramatically increased pharyngeal pumping (data not shown). The inhibition of mammalian voltage-gated A-type potassium channels by PKA (Schrader et al., 2002) and the expression of shl-1 in MC (Fawcett et al., 2006) suggest the possibility that $\mathrm{G}_{\mathrm{s}} \alpha$ signaling may stimulate pharyngeal pumping by inhibiting SHL-1. Another interesting question to be solved is why SER-7 in $\mathrm{MC}$ and M4 activated the two neurons mainly by activating two distinct G-protein signaling cascades. It may be because components of the $G_{12} \alpha$ signaling pathway are not expressed in MC. It is notable that expression of constitutively active GPA-12 or
RHO-1 in cholinergic neurons did not alter pharyngeal pumping rate (Fig. $3 B, C$ ).

We also found that, despite their separate neuronal regulation, isthmus peristalsis was coupled to the preceding pump. How are the two feeding motions coupled? A previous calcium imaging study reported that an anterior-to-posterior calcium wave travels in the posterior isthmus selectively during isthmus peristalsis (Shimozono et al., 2004), suggesting that the calcium wave dictates the muscle contraction. When a pump occurs, the electrical signal that excites the pharyngeal muscles to elicit pumping travels from the corpus to the terminal bulb (Raizen and Avery, 1994; Shimozono et al., 2004). The observations that M4 killing results in failure of isthmus peristalsis (Avery and Horvitz, 1987) and that peristalsis requires pumping suggest that induction of the calcium wave that causes peristalsis requires both muscle excitation during pumping and stimulation by M4 (Fig. $5 B, C$ ). This model explains how the feeding motions can be separately regulated yet coupled.

The pharynx may have evolved to regulate feeding motions in this way to support efficient feeding. Separate regulation of the two feeding rates allows worms to adjust the ratio of isthmus peristalsis to pharyngeal pumping (IP-to-PP ratio) according to the density of food. When food is scarce, the IP-to-PP ratio would decrease because worms need to take in food as actively as possible, but they do not need to swallow frequently. When food is abundant, however, the ratio would increase because worms do not need to work so hard to take in food, but they need to swallow frequently so that the anterior pharynx does not become full. In fact, we observed that the average IP-to-PP ratio tends to be higher when the pharynx pumps slowly (Fig. 1D). Furthermore, the regulators of feeding that we found can be classified into two groups: The first group, such as components of the $\mathrm{G}_{s} \alpha$ or $d g k-1$ signaling pathways, increases both feeding rates when activated or inhibited. The second group, such as gpa-12 (Fig. $3 E, F$ ), pkc-1 (data not shown), and unc-31 (Fig. 4F), decreased isthmus peristalsis but increased pharyngeal pumping when suppressed. To adjust the IP-to-PP ratio according to food density, activity of the second group may be selectively suppressed when food is scarce.

The coupling of the two feeding motions also supports efficient feeding by coordinating them. Food accumulated by pumping is transported from the anterior isthmus to the grinder in the terminal bulb by isthmus peristalsis. Thus, isthmus peristalsis would be futile in the absence of pumping and the coupling of isthmus peristalsis to the preceding pump may serve to prevent such futile feeding motions.

Several interesting questions remain to be answered. For instance, by what mechanisms are the frequency and timing of onset of the feeding motions determined? How does a single isthmus muscle show two different feeding motions? Further studies to answer these questions may lead us to understand how nervous system and muscle interact for sophisticated regulation of muscle motions.

\section{References}

Albertson DG, Thomson JN (1976) The pharynx of Caenorhabditis elegans. Philos Trans R Soc Lond B Biol Sci 275:299-325.

Alfonso A, Grundahl K, Duerr JS, Han HP, Rand JB (1993) The Caenorhabditis elegans unc-17 gene: a putative vesicular acetylcholine transporter. Science 261:617-619.

Aravamudan B, Broadie K (2003) Synaptic Drosophila UNC-13 is regulated by antagonistic G-protein pathways via a proteasome-dependent degradation mechanism. J Neurobiol 54:417-438.

Avery L, Horvitz HR (1987) A cell that dies during wild-type C. elegans 
development can function as a neuron in a ced-3 mutant. Cell 51:1071-1078.

Avery L, Horvitz HR (1989) Pharyngeal pumping continues after laser killing of the pharyngeal nervous system of C. elegans. Neuron 3:473-485.

Barsh GS, Schwartz MW (2002) Genetic approaches to studying energy balance: perception and integration. Nat Rev Genet 3:589-600.

Berthoud HR (2004) Neural control of appetite: cross-talk between homeostatic and non-homeostatic systems. Appetite 43:315-317.

Brenner S (1974) The genetics of Caenorhabditis elegans. Genetics 77:71-94.

Chiang JT, Steciuk M, Shtonda B, Avery L (2006) Evolution of pharyngeal behaviors and neuronal functions in free-living soil nematodes. J Exp Biol 209:1859-1873

Croll NA (1978) Integrated behavior in the feeding phase of Caenorhabditis elegans. J Zool 184:507-517.

Douglas SJ, Dawson-Scully K, Sokolowski MB (2005) The neurogenetics and evolution of food-related behaviour. Trends Neurosci 28:644-652.

Fawcett GL, Santi CM, Butler A, Harris T, Covarrubias M, Salkoff L (2006) Mutant analysis of the Shal (Kv4) voltage-gated fast transient $\mathrm{K}^{+}$channel in Caenorhabditis elegans. J Biol Chem 281:30725-30735.

Hiley E, McMullan R, Nurrish SJ (2006) The Galpha12-RGS RhoGEFRhoA signalling pathway regulates neurotransmitter release in C. elegans. EMBO J 25:5884-5895.

Hobert O (2002) PCR fusion-based approach to create reporter gene constructs for expression analysis in transgenic C. elegans. Biotechniques 32: $728-730$.

Hobson RJ, Geng J, Gray AD, Komuniecki RW (2003) SER-7b, a constitutively active Galphas coupled 5- $\mathrm{HT}_{7}$-like receptor expressed in the Caenorhabditis elegans M4 pharyngeal motorneuron. J Neurochem 87:22-29.

Hobson RJ, Hapiak VM, Xiao H, Buehrer KL, Komuniecki PR, Komuniecki RW (2006) SER-7, a Caenorhabditis elegans 5- $\mathrm{HT}_{7}$-like receptor, is essential for the 5-HT stimulation of pharyngeal pumping and egg laying. Genetics 172:159-169.

Horvitz HR, Chalfie M, Trent C, Sulston JE, Evans PD (1982) Serotonin and octopamine in the nematode Caenorhabditis elegans. Science 216:1012-1014.

Jose AM, Koelle MR (2005) Domains, amino acid residues, and new isoforms of Caenorhabditis elegans diacylglycerol kinase 1 (DGK-1) important for terminating diacylglycerol signaling in vivo. J Biol Chem 280:2730-2736.

Kaun KR, Sokolowski MB (2009) cGMP-dependent protein kinase: linking foraging to energy homeostasis. Genome 52:1-7.

Kim K, Li C (2004) Expression and regulation of an FMRFamide-related neuropeptide gene family in Caenorhabditis elegans. J Comp Neurol 475:540-550.

Kvachnina E, Liu G, Dityatev A, Renner U, Dumuis A, Richter DW, Dityateva
G, Schachner M, Voyno-Yasenetskaya TA, Ponimaskin EG (2005) $5-\mathrm{HT}_{7}$ receptor is coupled to $\mathrm{G} \alpha$ subunits of heterotrimeric G12-protein to regulate gene transcription and neuronal morphology. J Neurosci 25:7821-7830.

McKay JP, Raizen DM, Gottschalk A, Schafer WR, Avery L (2004) eat-2 and eat-18 are required for nicotinic neurotransmission in the Caenorhabditis elegans pharynx. Genetics 166:161-169.

McMullan R, Hiley E, Morrison P, Nurrish SJ (2006) Rho is a presynaptic activator of neurotransmitter release at pre-existing synapses in C. elegans. Genes Dev 20:65-76.

Raizen DM, Avery L (1994) Electrical activity and behavior in the pharynx of Caenorhabditis elegans. Neuron 12:483-495.

Raizen DM, Lee RY, Avery L (1995) Interacting genes required for pharyngeal excitation by motor neuron MC in Caenorhabditis elegans. Genetics 141:1365-1382.

Rand JB, Russell RL (1984) Choline acetyltransferase-deficient mutants of the nematode Caenorhabditis elegans. Genetics 106:227-248.

Reynolds NK, Schade MA, Miller KG (2005) Convergent, RIC-8-dependent Galpha signaling pathways in the Caenorhabditis elegans synaptic signaling network. Genetics 169:651-670.

Richmond JE, Weimer RM, Jorgensen EM (2001) An open form of syntaxin bypasses the requirement for UNC-13 in vesicle priming. Nature 412:338-341.

Schafer WR (2005) Deciphering the neural and molecular mechanisms of $C$. elegans behavior. Curr Biol 15:R723-R729.

Schrader LA, Anderson AE, Mayne A, Pfaffinger PJ, Sweatt JD (2002) PKA modulation of Kv4.2-encoded A-type potassium channels requires formation of a supramolecular complex. J Neurosci 22:10123-10133.

Shimozono S, Fukano T, Kimura KD, Mori I, Kirino Y, Miyawaki A (2004) Slow $\mathrm{Ca}^{2+}$ dynamics in pharyngeal muscles in Caenorhabditis elegans during fast pumping. EMBO Rep 5:521-526.

Sieburth D, Madison JM, Kaplan JM (2007) PKC-1 regulates secretion of neuropeptides. Nat Neurosci 10:49-57.

Sze JY, Victor M, Loer C, Shi Y, Ruvkun G (2000) Food and metabolic signalling defects in a Caenorhabditis elegans serotonin-synthesis mutant. Nature 403:560-564.

You YJ, Kim J, Raizen DM, Avery L (2008) Insulin, cGMP, and TGF-beta signals regulate food intake and quiescence in C. elegans: a model for satiety. Cell Metab 7:249-257.

Zheng H, Berthoud HR (2008) Neural systems controlling the drive to eat: mind versus metabolism. Physiology 23:75-83.

Zhou KM, Dong YM, Ge Q, Zhu D, Zhou W, Lin XG, Liang T, Wu ZX, Xu T (2007) PKA activation bypasses the requirement for UNC-31 in the docking of dense core vesicles from C. elegans neurons. Neuron 56:657669. 